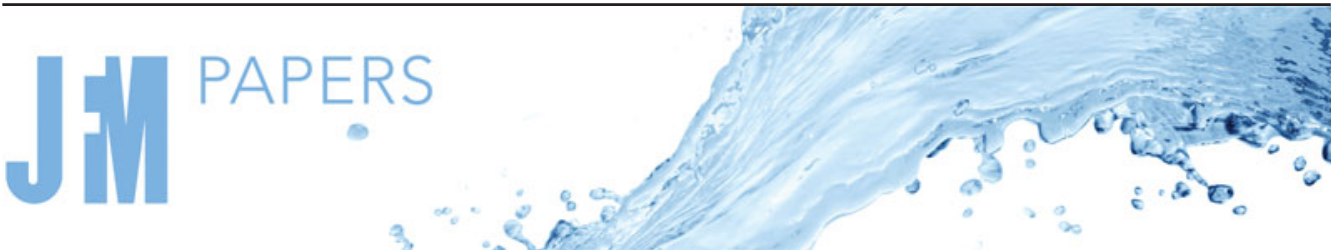

\section{On the turbulent flow past a realistic open-cell metal foam}

\author{
R. Corsini ${ }^{1}$, A. Fregni ${ }^{1}$, M. Spinolo ${ }^{2}$ and E. Stalio ${ }^{1}{ }^{\dagger}$ \\ ${ }^{1}$ Dipartimento di Ingegneria “Enzo Ferrari”, Università degli Studi di Modena e Reggio Emilia, via Pietro \\ Vivarelli 10, 41125 Modena, Italy \\ ${ }^{2}$ Ferrari S.p.A., via Enzo Ferrari 27, 41053 Maranello, Italy
}

(Received 30 July 2020; revised 19 March 2021; accepted 11 May 2021)

Turbulence is investigated in the lee of an open-cell metal foam layer. In contrast to canonical grids, metal foams are locally irregular but statistically isotropic. The solid matrix is characterised by two lengths, the ligament thickness $d_{f}$ and the pore diameter $d_{p}$. A direct numerical simulation is conducted on a realistic metal foam geometry for which $d_{f} / d_{p}=0.14$ and the porous layer thickness is five times the pore diameter. The Reynolds number based on the pore size is $R e_{d_{p}}=4000$, corresponding to a Taylor-scale Reynolds number $R e_{\lambda} \approx 80$. Closer to the foam than two pore diameters, the pressure and turbulent transports of turbulent kinetic energy are non-negligible. In the same region, $R e_{\lambda}$ undergoes a steep decrease whereas the dissipation coefficient $C_{\epsilon}$ increases like $R e_{\lambda}^{-1}$. At larger distances from the porous layer, the classical grid turbulence situation is recovered, where the mean advection of turbulent kinetic energy equals dissipation. This entails a power-law decay of turbulent quantities and characteristic lengths. The decaying exponents of integral, Taylor and Kolmogorov scales are close to one-half, indicating that the turbulence simulated here differs from Saffman turbulence. Analysis of the scaling exponents of structure functions and the decorrelation length of dissipation reveals that small-scale fluctuations are weakly intermittent.

Key words: isotropic turbulence, homogeneous turbulence

\section{Introduction}

Grid turbulence has been investigated for a long time owing to its good approximation to homogeneous and isotropic turbulence. In the classic flow configuration, a planar grid or

$†$ Email address for correspondence: enrico.stalio@unimore.it

(C) The Author(s), 2021. Published by Cambridge University Press. This is an Open Access article, distributed under the terms of the Creative Commons Attribution licence (http://creativecommons.org/

licenses/by/4.0/), which permits unrestricted re-use, distribution, and reproduction in any medium, provided the original work is properly cited. 


\section{R. Corsini, A. Fregni, M. Spinolo and E. Stalio}

screen with uniform mesh size and definite rod thickness is held in a uniform fluid stream. Downstream of the grid, homogeneity is achieved on cross-flow planes and a degree of isotropy is exhibited. This canonical flow has assumed an essential role in understanding turbulence and has allowed the formulation and testing of theories and models since the early wind tunnel experiments (Simmons \& Salter 1934; Taylor 1935).

The search for self-preserving or self-similar forms of correlation or spectral functions has led to the theories of homogeneous isotropic turbulence proposed over the past decades and the common prediction that turbulence decays according to a power law (de Karman \& Howarth 1938; Kolmogorov 1941; Batchelor 1948; Saffman 1967; George 1992). Despite the fact that these theories agree on the form, the value of the decay exponent $n$ is still debated. The theoretical analyses by de Karman \& Howarth (1938), Batchelor (1948) and Saffman (1967) resulted in values for $n$ of $1,10 / 7$ and 6/5, respectively. A large collection of experimental measurements involving classical grids of various geometries and at different flow regimes has grown up over the years. While the earliest works supported the prediction that $n=1$ (Batchelor \& Townsend 1948), later experiments by Comte-Bellot \& Corrsin (1966) and Mohamed \& Larue (1990) corrected the decay exponent, with values falling between 1.1 and 1.4 (Lavoie, Djenidi \& Antonia 2007; Kurian \& Fransson 2009; Kitamura et al. 2014, and references therein). George (1992) argued that the apparent discrepancies in the measurements are related to an undefined dependence of the flow on the initial conditions. This precludes the existence of a single universal state, at least at finite Reynolds number. Lavoie et al. (2007) investigated the effects of the initial conditions on the characteristics of decaying turbulence, and showed that this impact is more marked as the anisotropy and the strength of large-scale periodic motions increase. On the other hand, the improvement of isotropy and of large-scale periodic character reduces the influence of initial conditions. This suggested that the dependence on initial conditions is associated with departures from ideal homogeneity and isotropy conditions.

Recent research has attempted to reconcile many of the experimental data with Saffman turbulence. According to these analyses, the value $n=6 / 5$ represents a minimum decay rate valid for (strictly) homogeneous turbulence and which could also have deviations because of an inhomogeneous decay. The large-scale turbulent structures are proven to be regulated by the invariance of the Saffman integral. This is physically interpreted as the conservation of linear momentum and is opposed to the invariance of the Loitsyansky integral, on which Batchelor turbulence is based. It is concluded in the work by Krogstad \& Davidson (2010) that 'it seems very likely' that the turbulence observed is Saffman turbulence, and that it is also possible that different grid geometries, or other ranges of $R e$, could produce different results. Also Kitamura et al. (2014) report that grid turbulence is of Saffman type for the Reynolds-number range examined in their work, when grid turbulence is generated by a square or a cylindrical grid.

Aside from the existence of a self-preserving solution, Mohamed \& Larue (1990) attributed the difficulty in finding a single decay exponent also to inconsistencies in the data analysis method. In particular, the inaccuracy is related to the uncertainty in the virtual origin and the inclusion of data pertaining to the inhomogeneous and anisotropic region in the developed range. For grid turbulence, the turbulence decay is to be evaluated starting from $x / M>40-60$, where $x$ is the distance from the grid and $M$ is the mesh width.

A class of turbulence-generating grid consisting of fractal geometries has been introduced starting from the work by Hurst \& Vassilicos (2007). Since then, fractal 


\section{Turbulent flow past a metal foam}

grids have attracted a lot of interest because of the specific type of turbulence generated. A remarkable increase in the Reynolds number based on the Taylor scale with respect to usual passive grids was noticed by Seoud \& Vassilicos (2007). Further studies investigating the decaying turbulence downstream of a set of multiscale grids (Krogstad \& Davidson 2012) and a square-element fractal grid (Hearst \& Lavoie 2014) have revealed that, while the region close to the grids can be characterised by residual inhomogeneity and is grid-dependent, in the far field - where development is accomplished - flow characteristics are in accordance with classical grid turbulence measurements.

The in-depth study of turbulence generated by fractal grids has revealed a peculiar behaviour of the dissipation coefficient $C_{\epsilon}=\langle\epsilon\rangle L / u^{3}$ (here $\langle\epsilon\rangle$ is the rate of dissipation.) in a region close to the turbulence-generating grid but in conjunction with energy spectra, which follow the $-5 / 3$ slope for a wide range of wavenumbers. This behaviour has been described as a breakdown of the classical dissipation scaling and is observed in wind tunnel experiments of grid-generated turbulence of different geometries (Mazellier \& Vassilicos 2010; Isaza, Salazar \& Warhaft 2014; Valente \& Vassilicos 2014; Mora et al. 2019) and also in direct numerical simulation (DNS) data of decaying turbulence in a periodic box (Goto \& Vassilicos 2016). The breakdown consists in a behaviour of $C_{\epsilon}$ at the initial stages of the decay that depends upon the inlet Reynolds number $R e_{M}$ and the local Reynolds number $R e_{\lambda}$ as follows: $C_{\epsilon} \sim \operatorname{Re}_{M}^{1 / 2} / \operatorname{Re}_{\lambda}$. This implies that $L / \lambda \sim R e_{M}^{1 / 2}$ along the direction of $R e_{\lambda}$ decay (Valente \& Vassilicos 2012).

The turbulent flow behind an open-cell metal foam has never been investigated before. Similar to regular grids, the open-cell metal foam geometry is characterised by two main length scales, i.e. the mean pore diameter $d_{p}$ and the mean ligament thickness $d_{f}$. In contrast to regular grids, open cells are arranged randomly in space and their morphology, based on a polyhedral frame, is never exactly repeated. This generates a structure that is highly irregular and anisotropic at the pore scale but statistically isotropic at the macroscale. In addition, the metal foam layer investigated here has a thickness larger than a single ligament or a pore. Moreover, the solidity of metal foams, measured as $1-\varepsilon$, where $\varepsilon$ represents grid porosity, is very different from the solidities typical of grids employed for grid turbulence, which generally range between 0.30 and 0.45 . In high-porosity metal foams, this value is typically lower than 0.10 (Calmidi \& Mahajan 2000).

In this paper, a description is reported of turbulence downstream of a high-porosity open-cell metal foam. After a qualitative introduction to the flow investigated in $\S 3.1$, the degree of homogeneity and isotropy of turbulence is investigated in $\S 3.2$. The power-law behaviour of turbulent kinetic energy $\langle k\rangle$ and its dissipation rate are assessed in $\S 3.3$ and $\S 3.4$, respectively. The streamwise variations of turbulent length scales are considered in $\S 3.5$. In $\S 3.6$ the behaviour of the dissipation rate coefficient is investigated in detail, also in the context of recent research on the topic. In $\S 3.7$ the discussion is about whether or not Saffman turbulence is achieved for the present flow configuration. The multiscale nature of the flow is examined by means of one-dimensional spectra in $\S 3.8$. Then $\S 3.9$ investigates high-order statistics, the role of intermittency and the decorrelation length of dissipation. Budget terms of turbulent kinetic energy and of velocity variances are reported in $\S 3.10$, where the main mechanisms for the transport of energy and fluctuations are described in the vicinity of the solid structure and in the fully developed region. Final remarks are drawn in $\S 4$. 


\section{Numerical methodology}

\subsection{Mathematical formulation and flow configuration}

The system of equations solved numerically comprises the mass conservation and the Navier-Stokes equations for incompressible flows:

$$
\left\{\begin{array}{l}
\frac{\partial U_{i}}{\partial x_{i}}=0 \\
\frac{\partial U_{i}}{\partial t}+\frac{\partial U_{i} U_{j}}{\partial x_{j}}=-\frac{\partial P}{\partial x_{i}}+\frac{1}{R e_{d_{p}}} \frac{\partial^{2} U_{i}}{\partial x_{j} \partial x_{j}},
\end{array}\right.
$$

complemented with appropriate boundary conditions. The subscripts $i$ and $j$ take the values 1, 2 and 3 to denote the streamwise direction, $x$, and the two cross-flow directions, $y$ and $z$, respectively. All the variables are made non-dimensional by the velocity at the inlet $U_{\infty}$ and the mean pore diameter of the metal foam $d_{p}$. The Reynolds number based on the unperturbed velocity and the mean pore diameter is $R e_{d_{p}}=4000$.

The governing equations (2.1) are solved using the high-order finite-difference method implemented in Incompact3d (Laizet \& Lamballais 2009). Sixth-order compact schemes are used for spatial discretisation (Lele 1992), and time integration is done by the third-order Adams-Bashforth scheme. The velocity field is evaluated on a Cartesian grid with uniform spacing along the three directions, and pressure is defined on a staggered grid. Pressure-velocity decoupling is accomplished by a fractional-step method, which determines the divergence-free velocity field by solving a Poisson equation. The Poisson problem is tackled in the spectral space by using a modified wavenumber formalism, which allows for any kind of boundary conditions for the velocity field in the physical space. Inflow/outflow boundary conditions are enforced along the streamwise direction and periodic boundary conditions are set along the cross-flow directions to represent statistical homogeneity. A uniform velocity field is prescribed at the inlet $\left(U_{\infty}, 0,0\right)$, while at the outlet the velocity is determined by a convection equation:

$$
\frac{\partial U_{i}}{\partial t}+c \frac{\partial U_{i}}{\partial x}=0 .
$$

The convection velocity $c$ is calculated at each time step as the mean between the maximum and minimum values of the streamwise velocity component at the outlet.

The representation of the intricate metal foam geometry is achieved via an immersed boundary method (IBM) based on direct forcing (Gautier, Laizet \& Lamballais 2014). The IBM enforces the no-slip condition at the solid walls while preserving the simplicity of the finite-difference schemes applied to the Cartesian grid (Laizet \& Lamballais 2009). Further details on the numerical methodology employed here are provided in Corsini \& Stalio (2020).

A sketch of the computational domain is displayed in figure 1. The extents of the domain along the streamwise and the two cross-flow directions are $L_{x}=45 d_{p}$ and $L_{y}=L_{z}=$ $11.25 d_{p}$, respectively. The origin of the coordinate system is located at the centre of the downstream face of the porous matrix; thus $x=0$ describes the most upstream cross-flow plane where the fluid is not in contact with the solid phase. The thickness of the metal foam layer in the streamwise direction is $5 d_{p}$ and it spans the whole domain in the cross-flow directions. It is placed at a $5 d_{p}$ distance from the inlet section; this avoids interference between the upstream boundary and the solid matrix. 


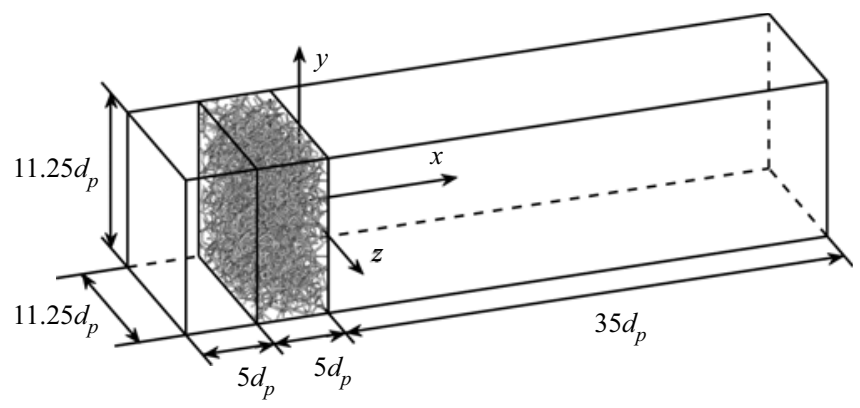

Figure 1. Schematic representation of the computational domain.

The computational domain is discretised by $n_{x}=3073$ grid nodes in the streamwise direction and $n_{y}=n_{z}=768$ in the cross-flow directions. The spatial resolution is sufficiently fine to ensure that $\Delta x=\Delta y=\Delta z \leqslant 2 \eta$ for $x \geqslant 5$. Close to the porous layer $(0<x<5)$, where dissipation is larger, $\Delta x=\Delta y=\Delta z \leqslant 5 \eta$. In the above comparisons, the Kolmogorov microscale $\eta$ is calculated a posteriori from its definition. The time step is kept at $\Delta t=0.001 d_{p} / U_{\infty}$ during the simulation, which in terms of the Kolmogorov time scale $\tau_{\eta}$ yields $\Delta t \leqslant 0.033 \tau_{\eta}$. This corresponds to a Courant-Friedrichs-Lewy number $C F L<0.3$.

Statistical quantities are computed by averaging in time and along the homogeneous $y$ and $z$ directions. Gathering of statistics begins after one flow-through time from the start of the simulation. In order to obtain well-converged statistics, the time interval of collection is $T=225 d_{p} / U_{\infty}$, and the three-dimensional snapshots of the velocity and pressure field are sampled at equal time intervals of $\Delta T=4.5 d_{p} / U_{\infty}$.

\subsection{Metal foam geometry}

The problem of the computer modelling of an intricate metal foam porous structure has been tackled in different ways. Pore-scale morphology can be reconstructed through X-ray tomography (Piller et al. 2013) or generated mathematically assuming an ideal cell geometry based on a virtual sample of regular polyhedra (Boomsma, Poulikakos \& Ventikos 2003). In this study, where geometric periodicity is a key feature of the numerical representation and irregularity of the foam is a requirement, a third approach is adopted: the open-pore cellular structure is generated synthetically through a numerical algorithm, developed by August et al. (2015). Besides the excellent realism and periodicity, one further favourable feature of synthetic metal foams is the possibility to tune their porosity and permeability. Thanks to a diffuse interface representation of the phase-field approach (August et al. 2015), the thickness of ligaments can be easily adjusted. Figure 2 shows the details of a couple of cells of the synthetic structure.

The synthetic metal foam structure used in the simulation is characterised by the geometrical features listed in table 1 . Both $d_{p}$ and $d_{f}$ are calculated by spatial averages and thus represent the mean pore diameter and the mean ligament thickness of the metal foam sample. The value of grid porosity set, $\varepsilon=0.92$, is representative of high-porosity metal foams (Calmidi \& Mahajan 2000). Based on typical sizes of open-cell aluminium foams, an inflow velocity of $U_{\infty}=15 \mathrm{~m} \mathrm{~s}^{-1}$ is obtained at $R e_{d_{p}}=4000$, assuming air at standard conditions as the working fluid. Table 1 reports experimental conditions from previous wind tunnel studies on regular planar grids. 


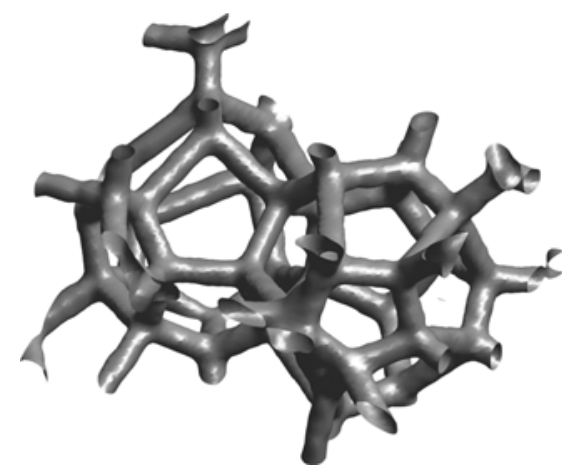

Figure 2. Cells of the aluminium foam generated algorithmically (August et al. 2015).

A sample of the metal foam geometry with superimposed computational points is displayed in figure 2 of Corsini \& Stalio (2020). Staircase patterns of the immersed boundaries approximate the rounded borders of the solid region. This referenced picture and the computed ratio between average ligament diameter and grid spacing $d_{f} / \Delta x \approx 10$ suggest that the ligaments are discretised by an adequate number of grid points.

\section{Results and discussion}

\subsection{Instantaneous and mean flows}

Figure 3(a) shows the streamwise component of the instantaneous velocity field in one of the snapshots collected. The uniform free stream of the inflow is disrupted by the irregularly arranged ligaments of the solid structure and velocity fluctuations arise within the porous matrix. Vortices of different orientations are shed from the ligaments and a wake is formed. The largest perturbations are observed close to the downstream edge of the porous matrix. The wakes originated by the ligaments develop in a non-uniform fashion and interact at variable lengths. The smaller wakes are seen to disappear after a couple of pore diameters, whereas larger wakes stemming from ligament clumps meet at a further distance from the foam. The larger wakes also last in time, as revealed by the streaks in the time-averaged velocity field $\langle U\rangle_{t}$ shown in figure $3(b)$.

\subsection{Homogeneity and isotropy}

The approximation to statistical homogeneity in the cross-flow directions in grid turbulence is known to depend on the grid geometry and the Reynolds number. While, for regular grids, experiments suggest that the flow becomes nearly homogeneous for $x / M>40$ (Comte-Bellot \& Corrsin 1966; Mohamed \& Larue 1990), for fractal grids, homogeneity is usually retrieved further downstream (Hearst \& Lavoie 2014) and, for example, Valente \& Vassilicos (2011) report $x / M_{\text {eff }}>80$, where $M_{\text {eff }}$ is the effective mesh size.

The distribution of $\langle U\rangle_{t}$ in cross-flow planes is shown in figure 4 for six streamwise positions at increasing distance from the metal foam. Solid lines mark regions where the percentage of variation of $\langle U\rangle_{t}$ relative to $U_{\infty}$ has magnitude greater than $10 \%$, while dashed lines encompass regions of magnitude greater than $5 \%$. These are seen to gradually shrink along $x$. While inhomogeneity is in part to be ascribed to the limited size of the sample composed only by the collection of snapshots in time, for $x>20$ their extent is 


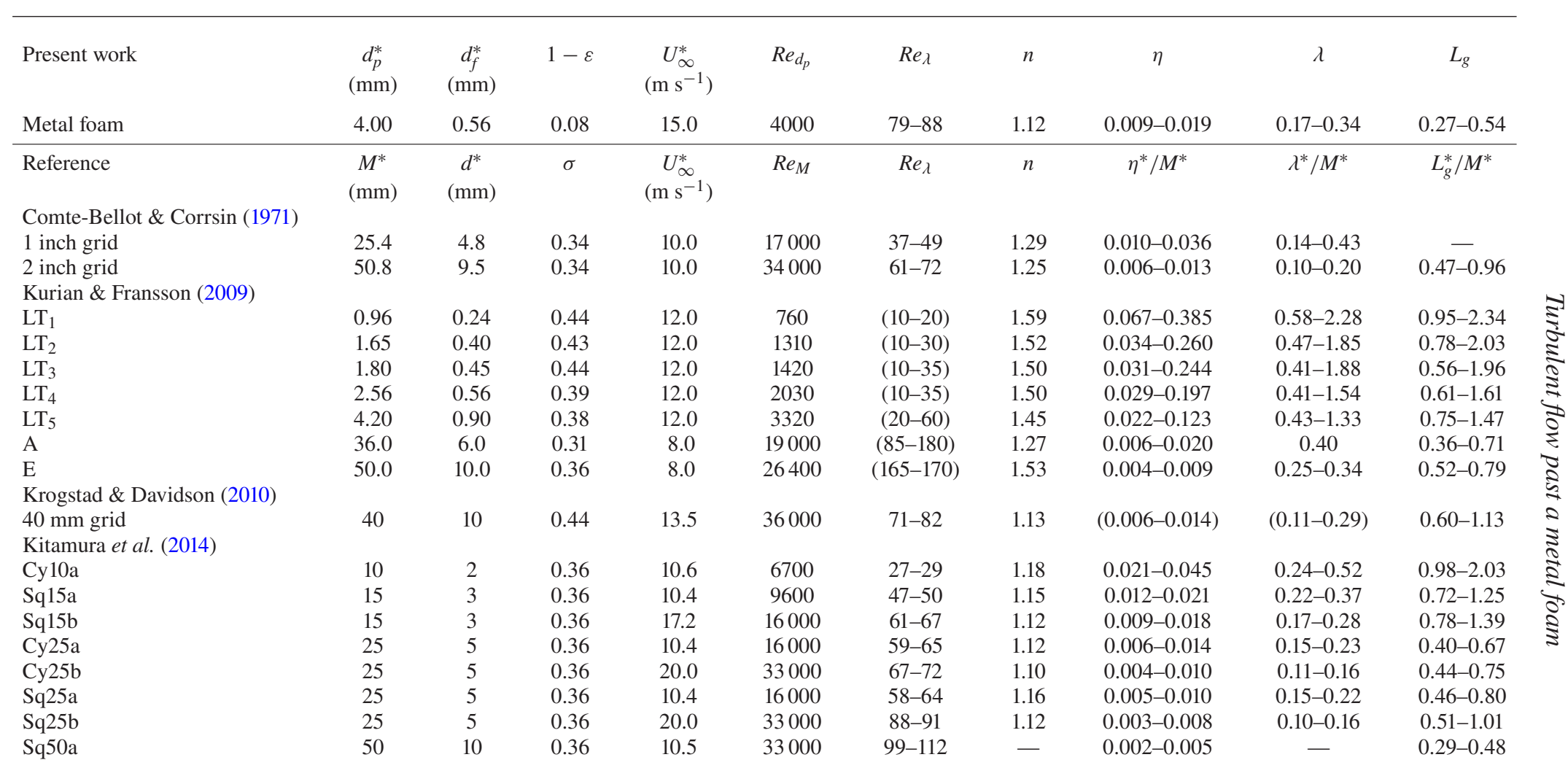

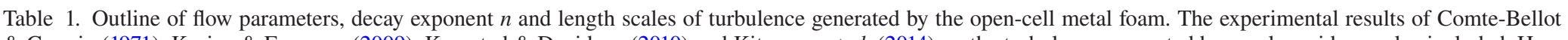

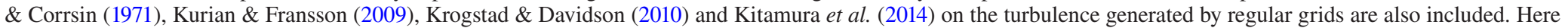

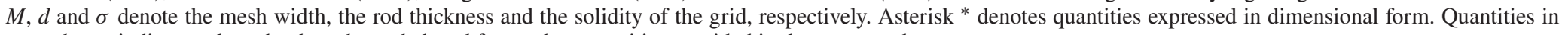
N parentheses indicate values that have been deduced from other quantities provided in the same work. 


\section{R. Corsini, A. Fregni, M. Spinolo and E. Stalio}
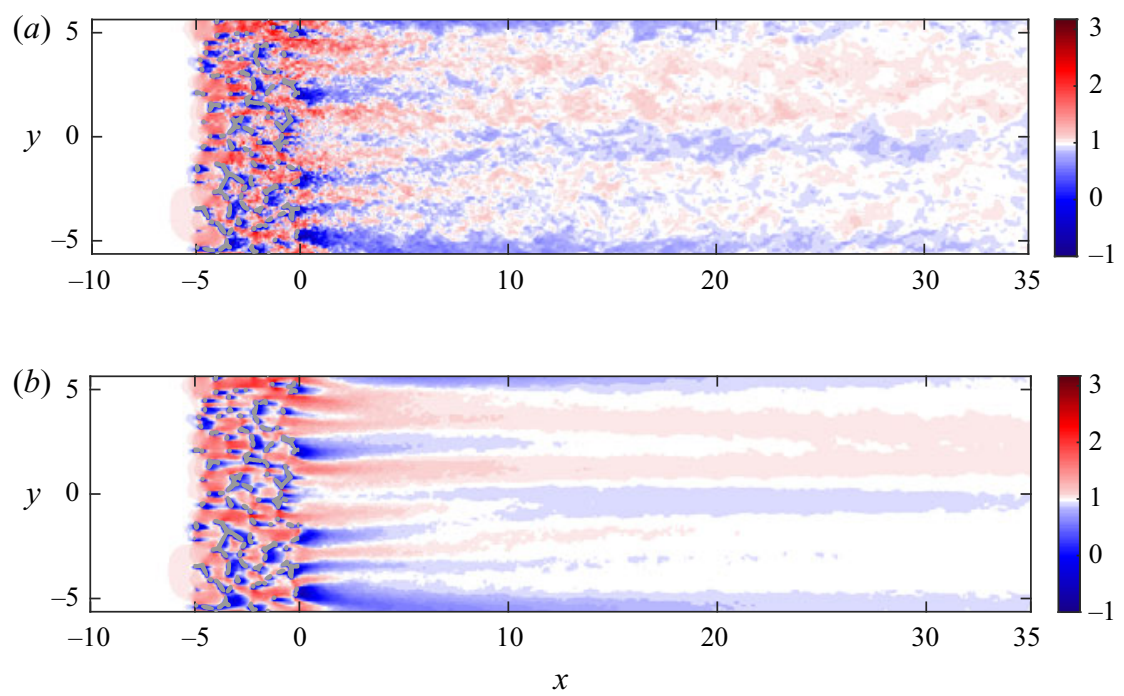

Figure 3. Instantaneous velocity field of the streamwise velocity component $(a)$ and its temporal mean $(b)$ in an $x-y$ plane.

still appreciable. In the $x=30$ station, the time-averaged velocity $\langle U\rangle_{t}$ varies between 0.86 and 1.12 .

The isotropy level of the large scales of motion can be investigated through the ratio of root-mean-square (r.m.s.) velocity fluctuations along orthogonal directions. In the present case, the fluctuations of the $x$-component of velocity are the largest: in the developed region, indicators $u_{r m s} / w_{r m s}$ and $u_{r m s} / v_{r m s}$ oscillate within the interval $(1.5,1.6)$ about a mean of 1.55 for $u_{r m s} / v_{r m s}$ and 1.56 for $u_{r m s} / w_{r m s}$, where the difference is finally due to the size of the sample employed in the simulations. In previous grid turbulence measurements (Kurian \& Fransson 2009; Krogstad \& Davidson 2010; Kitamura et al. 2014), the observed isotropy indicators are in general smaller than those measured here. Kitamura et al. (2014), who also collected experimental results by other authors, report $u_{r m s} / w_{r m s}<1.2$ in all cases; similar values are also reported in the lee of fractal grids (Hurst \& Vassilicos 2007; Gomes-Fernandes, Ganapathisubramani \& Vassilicos 2012). More details about large-scale isotropy measures downstream of the present metal foam are reported in Corsini \& Stalio (2020).

\subsection{Decay of velocity fluctuations}

Figure 5 displays the streamwise evolution of the variance of the three components of velocity $\left\langle u_{i} u_{i}\right\rangle$ as well as the turbulent kinetic energy $\langle k\rangle$. Very close to the foam and for $x<1$, velocity fluctuations are observed to remain constant. The negative slope increases gradually in $x$ until fluctuations exhibit a power-law decay that persists until the end of the computational domain. As demonstrated, for example, in Tennekes \& Lumley (1972), this is expected in the region where advection and dissipation of the turbulent kinetic energy become the only non-negligible terms in the transport equation of $\langle k\rangle$; see $\S 3.10$.

Power-law parameters are sought in the form

$$
\frac{u_{r m s}^{2}}{U_{\infty}^{2}}=A\left(\frac{x-x_{0}}{d_{p}}\right)^{-n}
$$




\section{Turbulent flow past a metal foam}
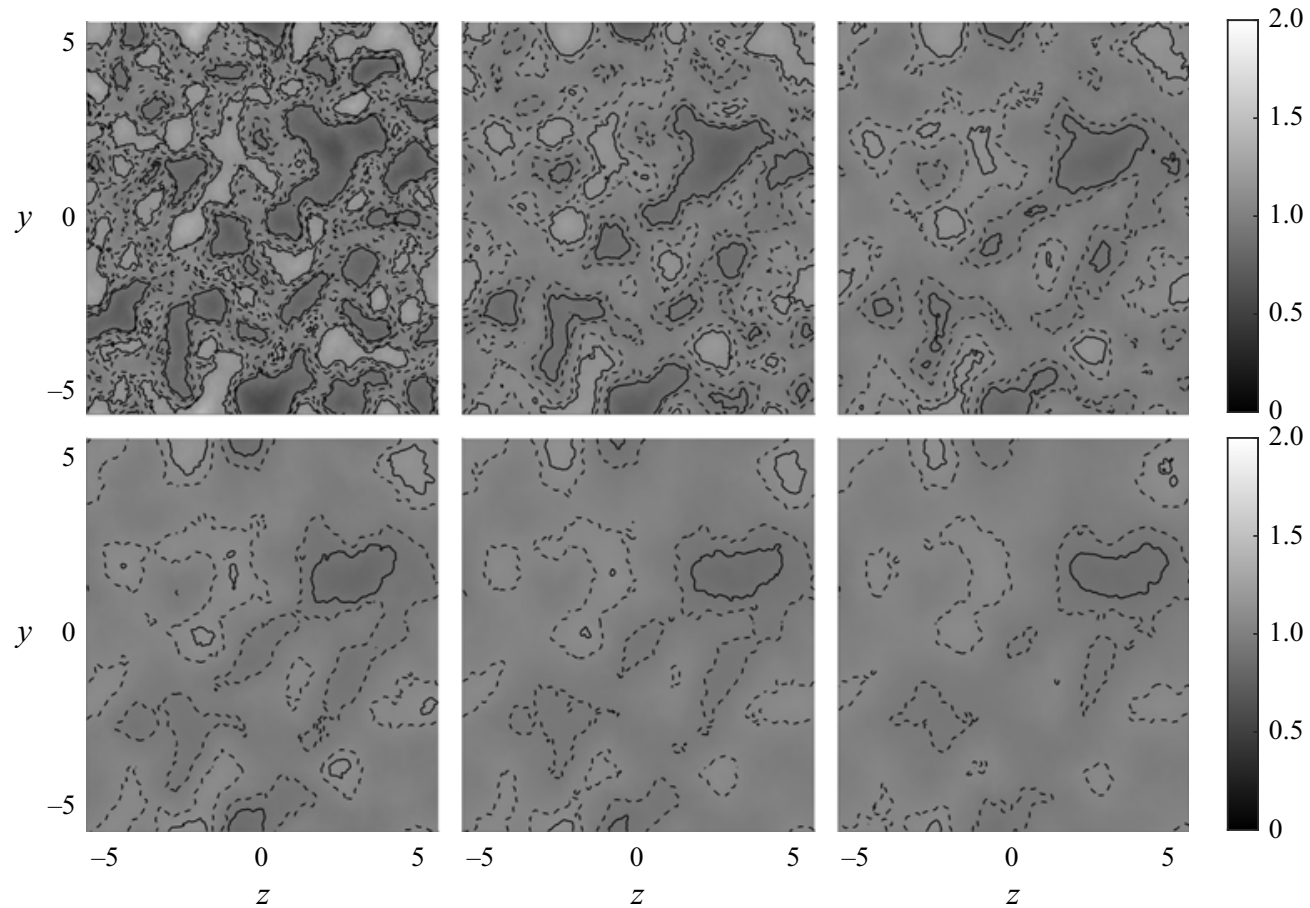

Figure 4. Streamwise mean flow field $\langle U\rangle_{t}$ in $y-z$ planes extracted at $x=5,10,15,20,25$ and 30 (left to right then top to bottom): solid line, isolines $\langle U\rangle_{t}-U_{\infty}= \pm 0.1$; and dashed line, isolines $\langle U\rangle_{t}-U_{\infty}= \pm 0.05$.

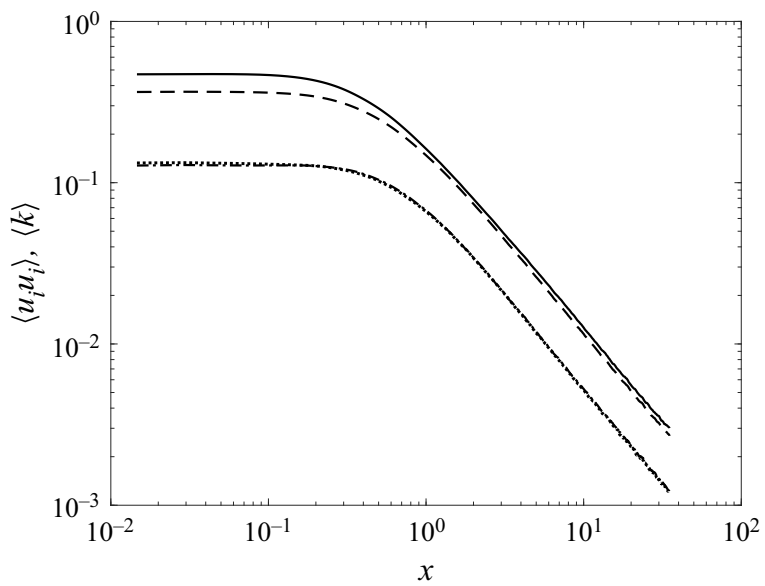

Figure 5. Decay of velocity fluctuations: solid line, $\langle u u\rangle$; dot-dashed line, $\langle v v\rangle$; dotted line, $\langle w w\rangle$; and dashed line, $\langle k\rangle$.

through a numerical procedure. In (3.1), $A$ is the multiplicative coefficient, $x_{0}$ is the virtual origin and $n$ is the decay exponent. As $n$ is positive, the power law has a vertical asymptote (and a singularity) at the virtual origin $x=x_{0}$. As the parameters in (3.1) depend greatly upon the interval of sampling data considered, also the interval limits are determined 


$\begin{array}{lccccc}\text { Variable } & x_{\min } & x_{\max } & x_{0} & A & n \\ u_{r m s}^{2} & 7.98 & 30.0 & 0.648 & 0.155 & 1.12 \\ \langle k\rangle & 6.83 & 30.0 & 0.310 & 0.155 & 1.14\end{array}$

Table 2. Left boundary of the fitting interval $I_{d}$, virtual origin, multiplicative coefficient and decay exponent of the power laws in the form of equation (3.1) fitting $u_{r m s}^{2}$ and $\langle k\rangle$.

inside the fitting procedure. A similar approach has been applied in Hearst \& Lavoie (2014).

In the present work, a developed region $I_{d}=\left[x_{\min }, x_{\max }\right]$ is employed, where the right border of the interval is kept fixed to $x_{\max }=30.0$, clear of possible - yet not evident outflow condition effects, while $x_{\min }$ is discretely varied in the interval $[0.015,24.7]$ to seek an $x_{\text {min }}$ coordinate that ensures the best fit. The coordinate $x_{\min }$ will be taken as the start of the developed region. For each selection of a value for $x_{\min }$, the virtual origin $x_{0}$ is discretely varied within $I_{0}=\left[0.015, x_{\min }\right]$, as the singularity is not supposed to belong to $I_{d}$. The intervals of variation of both $x_{\min }$ and $x_{0}$ are discretised by the same subdivision as the computational mesh. Parameters $A$ and $n$ are determined through a least-squares fit. Deviations between computed data and fitting laws are then calculated as the Euclidean norm of the error divided by the number of data points:

$$
e\left(x_{0}, x_{\text {min }}\right)=\frac{\left(\sum_{j=1}^{N_{d}} \delta_{j}^{2}\right)^{1 / 2}}{N_{d}} .
$$

In (3.2), $\delta_{j}$ represents the difference between computed statistics and the least-squares fitted power-law approximation of $u_{r m s}^{2}$ at the $j$ th point of $I_{d}$; and $N_{d}$ is the number of uniformly spaced points in the data fit region $I_{d}$. The $\left(x_{0}, x_{\min }\right)$ couple which ensures the smallest deviation from computed data provides the final $A$ and $n$ coefficients. This procedure leads to the results given in table 2 with error distribution as in figure 6 . In the region of parameters investigated, only one minimum is found, which is located far from the boundaries of the region investigated. The dependence on porosity of the power-law exponents obtained is shown to be only weak in the Appendix.

In order to check the dependence of the results from the fitting method employed, a procedure from the literature is also applied to the same set of data; this alternative technique is that utilised by Hearst \& Lavoie (2014). In the application of the method to the present case, the virtual origin $x_{0}$ and lower bound $x_{\min }$ are varied within $I_{0}=[0,4]$ and [4, 14.7], respectively. For both $u_{r m s}^{2}$ and $\langle k\rangle$, the process converges after three iterations. Applied to $u_{r m s}^{2}$, this fitting procedure provides $x_{0}=0.610$ and $x_{\min }=6.78$, which yield the estimate for $\tilde{n}_{u}=1.12$. In the case of $\langle k\rangle$, the values obtained are $x_{0}=0.360, x_{\text {min }}=$ 5.02 and $\tilde{n}_{k}=1.14$. The results obtained by the method proposed by Hearst \& Lavoie (2014) are only marginally different from those obtained as described above and reported in table 2.

Comte-Bellot \& Corrsin (1966) found $1.15 \leqslant n \leqslant 1.29$ for regular grids, while according to Mohamed \& Larue $(1990) n \approx 1.3$. More recently, Krogstad \& Davidson (2010) found $n=1.13 \pm 0.02$. 


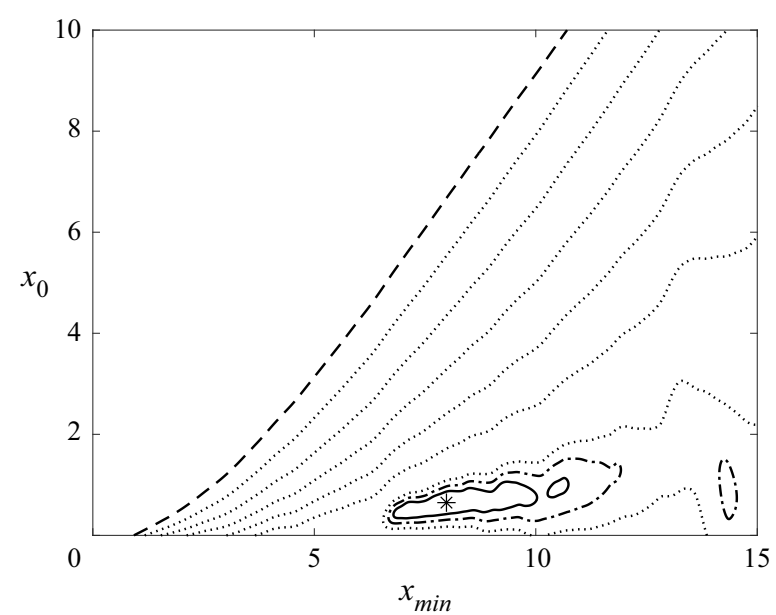

Figure 6. Contour lines of $\log _{10}(e)$, where $e$ is the normalised Euclidean norm of the error in (3.2): dashed line, $\log _{10}(e)=-4.8$; dot-dashed line, $\log _{10}(e)=-6.03$; solid line, $\log _{10}(e)=-6.05$; and dotted line, $\log _{10}(e) \in[-6.00-5.00]$, with lines separated by $\delta=0.2$. A single minimum is found in the field, as indicated by the $*$ symbol, which corresponds to $x_{0}=0.648$ and $x_{\min }=7.98$. The singularity is not included in the domain, and thus $x_{\min }>x_{0}$.

Besides the parameters $x_{0}, A$ and $n$ in (3.1), the procedure provides the coordinate $x_{\min }$ of the start of the developed region as the left boundary of the interval $I_{d}$. All the subsequent fittings in this work will be carried out on $I_{d}=[7.98,30.0]$.

\subsection{Turbulence decay rate}

As opposed to experimental studies, where rate of dissipation $\langle\epsilon\rangle$ needs to be evaluated using the frozen turbulence assumption or isotropy (3.5), in DNS studies $\langle\epsilon\rangle$ can be computed directly from its definition,

$$
\langle\epsilon\rangle \equiv \frac{2}{\operatorname{Re}_{d_{p}}}\left\langle s_{i j} s_{i j}\right\rangle
$$

where $s_{i j}=\left(\partial u_{i} / \partial x_{j}+\partial u_{j} / \partial x_{i}\right) / 2$ is the fluctuating rate of strain. Figure 7 displays the spatial evolution of $\langle\epsilon\rangle$, together with the least-squares fitted power law in the form $\langle\epsilon\rangle \sim a_{\epsilon} x^{h}$. The coefficients that fit the data over $I_{d}=[7.98,30.0]$ are $a_{\epsilon}=0.217$ and $h=-2.20$.

The scaling of dissipation $\langle\epsilon\rangle$ can also be set in relation to the scaling of $\langle k\rangle$ in $\S 3.3$. As also shown quantitatively in $\S 3.10$, in the developed region of a statistically steady, high-Reynolds-number flow, one has

$$
\langle U\rangle \frac{\mathrm{d} k}{\mathrm{~d} x}=-\langle\epsilon\rangle .
$$

Equation (3.4) suggests that the decay exponent in this case should equal $h=-\left(n_{k}+1\right)$. In the present study, $h=-2.20$ and $n_{k}=1.14$ are calculated. The percentage difference between $-\left(n_{k}+1\right)$ and $h$ is below $3 \%$. 


\section{R. Corsini, A. Fregni, M. Spinolo and E. Stalio}

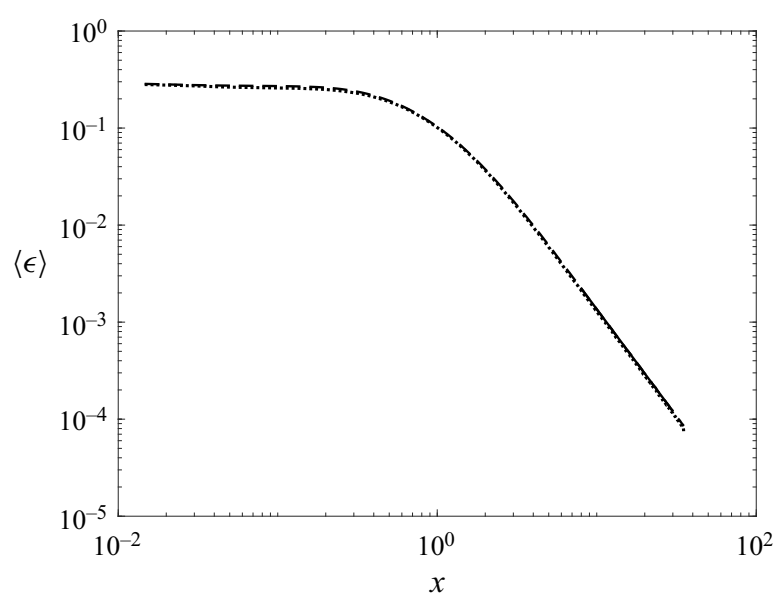

Figure 7. Dissipation of the turbulent kinetic energy: dashed line, $\langle\epsilon\rangle$ as defined in (3.3); dotted line, $\langle\epsilon\rangle_{\text {iso }}$ from (3.5); and solid line, $a_{\epsilon} x^{h}$, with $a_{\epsilon}=0.217$ and $h=-2.20$.

Dissipation is compared in figure 7 to the same quantity computed under the hypothesis of isotropic turbulence:

$$
\langle\epsilon\rangle_{\text {iso }}=15 v\left\langle\left(\frac{\partial u}{\partial x}\right)^{2}\right\rangle .
$$

The close similarity between $\langle\epsilon\rangle$ and $\langle\epsilon\rangle_{\text {iso }}$ is only in apparent contradiction with isotropy ratios larger than 1.5 reported in $\S 3.2$. From the demonstration by Taylor (1935), it appears that hypotheses less strict than isotropy are required for equation (3.5) to hold. The weaker set of hypotheses hold true in the present case; see Corsini \& Stalio (2020).

\subsection{Length scales}

The length scales examined for the characterisation of the turbulence generated by a metal foam are the Kolmogorov scale $\eta$, the Taylor microscale $\lambda$ and the integral scales. All the length scales are computed directly from their definitions. Their distribution within the developed region is approximated by a power law of the distance $x$. The range of variation of each length scale along $I_{d}$ is reported in table 1 together with data from the literature on classical grid turbulence.

\subsubsection{Kolmogorov scale}

The Kolmogorov scale is defined through dissipation $\langle\epsilon\rangle$. It is predicted from the power-law expression for $\langle\epsilon\rangle$ (derived from the expression for $\langle k\rangle$ ) that $\eta$ can be represented by a function of the form $\eta \sim a_{\eta} x^{s}$, where $s$ equals $-h / 4$ and finally $s=\left(n_{k}+1\right) / 4$. The decay exponent for $\langle k\rangle$ computed here, $n_{k}=1.14$, gives $s=0.54$. The power-law approximation is displayed in figure 8 together with the Kolmogorov scale, as computed from its definition: the fitting coefficients are $a_{\eta}=0.00291$ and $s=0.55$. Very similar power-law parameters are obtained in this same flow configuration for a porosity $\varepsilon=$ 0.97; see the Appendix. Comte-Bellot \& Corrsin (1971) provide Kolmogorov-scale measurements in their table 4 , which grow like a power law of exponent $\hat{s}=0.58$. 


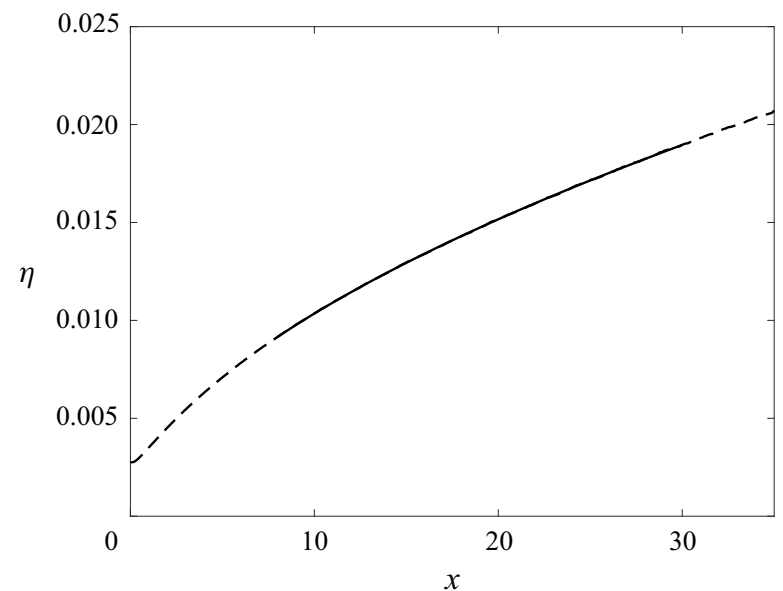

Figure 8. Streamwise distribution of the Kolmogorov scale: dashed line, $\eta$ as calculated from its definition; and solid line, $a_{\eta} x^{s}$, where $a_{\eta}=0.00291, s=0.55$. The uniform grid spacing adopted in all directions is $\Delta x_{i}=0.0146$, which suggests that spatial resolution is high enough to represent the smallest scales of the turbulence.

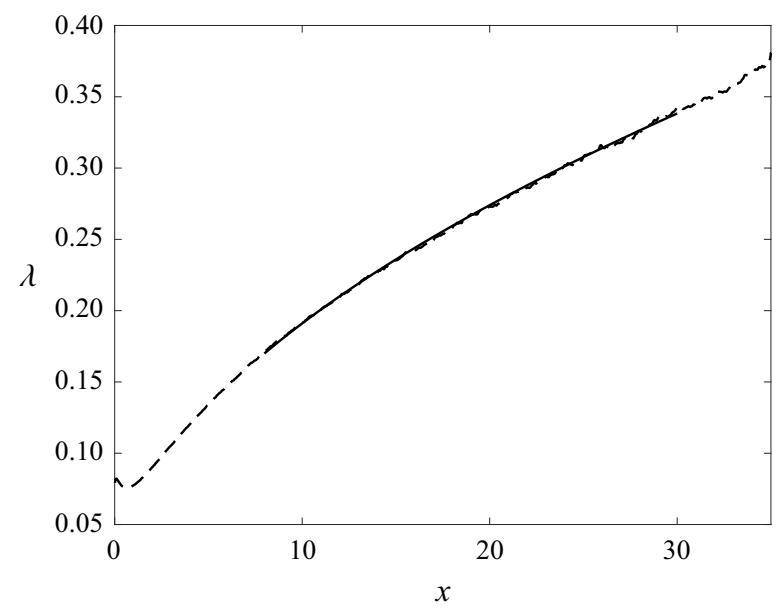

Figure 9. Streamwise distribution of the Taylor microscale: dashed line, $\lambda$ as calculated from its definition; and solid line, $a_{\lambda} x^{c}$, where $a_{\lambda}=0.0577$ and $c=0.52$.

\subsubsection{Taylor microscale}

Figure 9 displays the streamwise distribution of the Taylor microscale, defined by

$$
u_{r m s}^{2}=\lambda^{2}\left\langle\left(\frac{\partial u}{\partial x}\right)^{2}\right\rangle \text {. }
$$

Comte-Bellot \& Corrsin (1971) report Taylor-scale values in a few measurement points (see their table 4); their data fit a power law of exponent $c=0.53$. It is predicted in the study by George (1992) that the Taylor scale of homogeneous isotropic turbulence increases in time with $t^{1 / 2}$ and, for grid turbulence, $\lambda$ grows as $x^{1 / 2}$ in the laboratory frame. More recently, Kurian \& Fransson (2009) reported that $\lambda$ increases approximately like the square-root of the streamwise coordinate. In the present study, fitting a power-law approximation of the form $\lambda \sim a_{\lambda} x^{c}$ over $I_{d}$ gives $a_{\lambda}=0.0577$ and $c=0.52$. 


\section{R. Corsini, A. Fregni, M. Spinolo and E. Stalio}

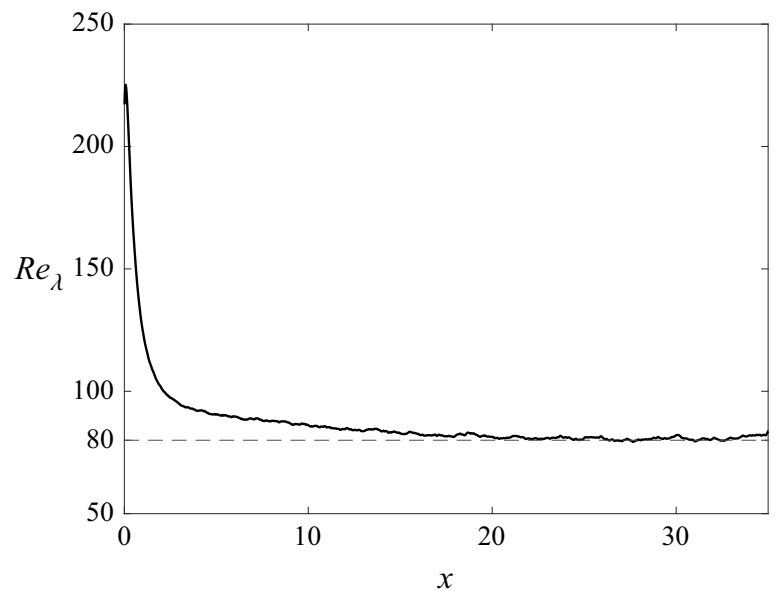

Figure 10. Streamwise distribution of $R e_{\lambda}$. The horizontal dashed line specifies the value $R e_{\lambda}=80$.

Figure 10 shows the behaviour of the turbulent Reynolds number. After a steep decrease in the vicinity of the metal foam, $R e_{\lambda}$ reaches a plateau where $R e_{\lambda} \approx 80$. An expression relating the Taylor and the Kolmogorov scales can be obtained for isotropic turbulence through (3.5):

$$
\frac{\lambda}{\eta}=15^{1 / 4} \operatorname{Re}_{\lambda}^{1 / 2}
$$

Results from the present investigation, and not reported for brevity, show that the difference between $(\lambda / \eta)^{2} / \sqrt{15}$ and $R e_{\lambda}$ is less than $3 \%$ over the whole computational domain.

\subsubsection{Integral scales}

The autocorrelation coefficient is defined as the ratio between the autocorrelation function of separation $\boldsymbol{r}=\boldsymbol{r} \boldsymbol{e}_{j}$ and the autocorrelation function for $r=0$ :

$$
\rho_{i i}(x, \boldsymbol{r})=\frac{R_{i i}(x, \boldsymbol{r})}{R_{i i}(x, 0)} .
$$

The autocorrelation coefficients along $y$ of the streamwise $\rho_{11}\left(x, r e_{2}\right)$ and the cross-flow $\rho_{22}\left(x, r \boldsymbol{e}_{2}\right)$ velocity components are reported in figure 8 of Corsini \& Stalio (2020). A distinction is made between the transverse and longitudinal correlations depending on the relative direction of velocity components and separation vector. Transverse correlations built with streamwise velocity fluctuations have longer tails with respect to longitudinal correlations built with spanwise fluctuations.

Integral scales are defined as integrals over $r$ of autocorrelation coefficients:

$$
L_{i i}\left(x, \boldsymbol{e}_{j}\right)=\frac{1}{R_{i i}(x, 0)} \int_{0}^{\infty} R_{i i}\left(x, r \boldsymbol{e}_{j}\right) \mathrm{d} r .
$$

These depend upon the coordinates along non-homogeneous directions as well as on the direction of separation $\boldsymbol{e}_{j}$. In practice, since the computational domain has finite boundaries, the integral scales are calculated here as the distance over which the autocorrelation function decreases from 1 to $1 / \mathrm{e}$, where e is Euler's number. For the calculation of integral scales, correlations are assumed to decay exponentially. 


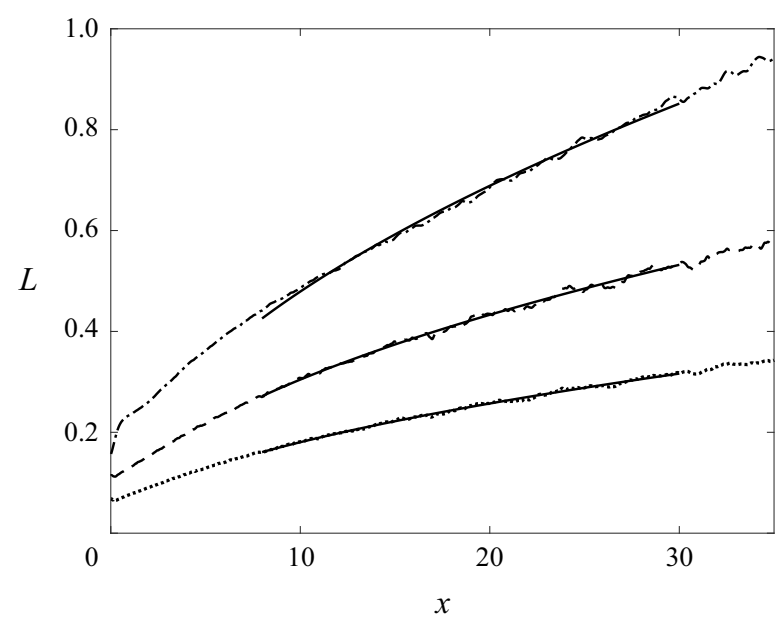

Figure 11. Streamwise distribution of the integral scales: dot-dashed line, streamwise integral scale $L$; dashed line, longitudinal integral scale $L_{g}$; dotted line, transverse integral scale $L_{t}$; and solid line, power-law approximations of the different scales.

$\begin{array}{lccr}\text { Variable } & \text { Power law } & \text { Multiplicative coefficient } & \text { Exponent } \\ \langle\epsilon\rangle(x) & a_{\epsilon} x^{h} & 0.217 & -2.20 \\ \eta(x) & a_{\eta} x^{s} & 0.00291 & 0.55 \\ \lambda(x) & a_{\lambda} x^{c} & 0.0577 & 0.52 \\ L(x) & a_{L} x^{q} & 0.143 & 0.52 \\ L_{g}(x) & - & 0.0946 & 0.51 \\ L_{t}(x) & - & 0.0555 & 0.51\end{array}$

Table 3. Parameters of the power-law functions of the form $f(x) \sim a x^{b}$ fitting the turbulent quantities analysed.

Integral scales for the present case are based on the streamwise or the cross-flow velocity components. Given the inhomogeneity of the streamwise direction, separation is set in the cross-flow directions $\boldsymbol{e}_{2}$ and $\boldsymbol{e}_{3}$. Thus, the integral scale based on the streamwise velocity is always transverse and will be denoted by $L$. The integral scales based on cross-flow velocity components can be either longitudinal or transverse and are denoted by $L_{g}$ and $L_{t}$, respectively. As statistically $L_{11}\left(x, \boldsymbol{e}_{2}\right)=L_{11}\left(x, \boldsymbol{e}_{3}\right)=L(x), L_{22}\left(x, \boldsymbol{e}_{2}\right)=L_{33}\left(x, \boldsymbol{e}_{3}\right)=$ $L_{g}(x)$ and $L_{33}\left(x, \boldsymbol{e}_{2}\right)=L_{22}\left(x, \boldsymbol{e}_{3}\right)=L_{t}(x)$, these equalities have been exploited in the calculation of integral scales.

Figure 11 displays the integral scales. The power-law approximations $L \sim a_{L} x^{q}$ have the coefficients reported in table 3; the exponents computed are very close to the $L \sim$ $x^{1 / 2}$ behaviour predicted by Wang \& George (2002). Also, in the case of higher porosity presented in the Appendix, the power-law exponent is close to $1 / 2$.

Notice that $L$ is one order of magnitude smaller than the domain size, the transverse length of which is $11.25 d_{p}$; this suggests that the imposed lateral periodic boundary conditions can satisfactorily represent cross-flow homogeneity in the present case. The evolution of the Reynolds number based on the integral scale, defined as $R e_{L} \equiv L u_{r m s} / \nu$, along the $x$-axis is displayed in figure 9 of Corsini \& Stalio (2020). 


\section{R. Corsini, A. Fregni, M. Spinolo and E. Stalio}

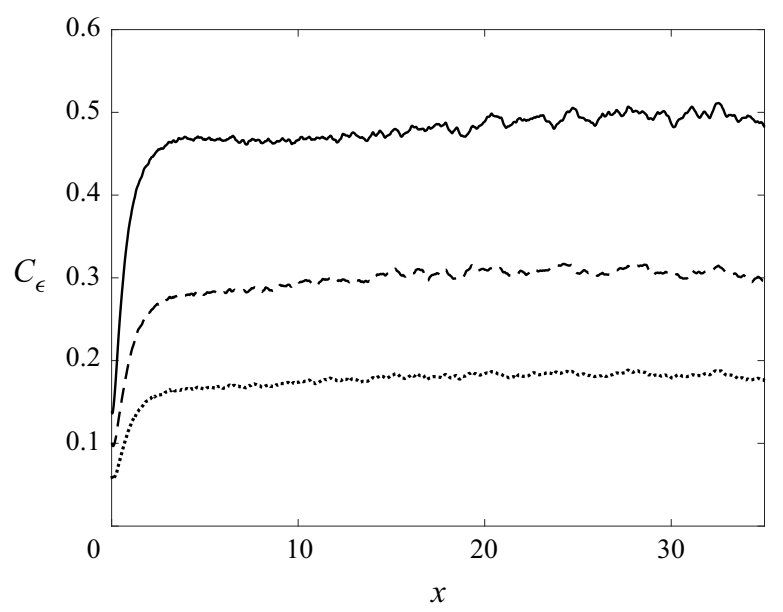

Figure 12. Streamwise distribution of $C_{\epsilon}=\langle\epsilon\rangle L / u_{r m s}^{3}$ : solid line, based on the streamwise integral scale $L$; dashed line, based on the longitudinal integral scale $L_{g}$; and dotted line, based on the transverse integral scale $L_{t}$.

\subsection{Dissipation rate coefficient}

In high-Reynolds-number turbulent flows away from solid walls, the dissipation rate can be scaled on the integral length scale and velocity fluctuations through an order-one constant:

$$
C_{\epsilon}=\frac{\langle\epsilon\rangle L}{u_{r m s}^{3}} .
$$

Figure 12 shows that, in the present case, after an initial steep increase for $x<2$, the dissipation rate coefficient $C_{\epsilon}$ based on $L$ fluctuates over $I_{d}$ between 0.45 and 0.50 , where its spatial average is $\bar{C}_{\epsilon}=0.483$. This value is very close to values reported in Pearson, Krogstad \& van de Water (2002) for shear turbulence at different $R e_{\lambda}$ numbers.

With regard to the initial steep increase, in recent years Vassilicos and coworkers have observed that, close to the turbulence-generating grid, there is a region characterised by spectra that closely match the $-5 / 3$ power law, in conjunction with an increase in $C_{\epsilon}$. In the hypothesis of $\langle\epsilon\rangle=\langle\epsilon\rangle_{i s o}$, combining the definition (3.10) with equation (3.5) leads to

$$
C_{\epsilon}=15 \frac{L}{\lambda} \frac{1}{R e_{\lambda}} .
$$

As only small variations of the ratio between length scales $L / \lambda$ are observed for given Reynolds number of the mesh size, $C_{\epsilon}$ is seen to increase like $\operatorname{Re}_{\lambda}^{-1}$. This behaviour is reported for both fractal and regular grids (Valente \& Vassilicos 2012).

Equation (3.11) is studied for the present case in figure 13, where the logarithmic plot emphasises the $C_{\epsilon}\left(R e_{\lambda}\right)$ behaviour close to the metal foam. Corresponding to the initial steep decrease in $R e_{\lambda}$ shown in figure $10, C_{\epsilon}$ is seen to increase like $R e_{\lambda}^{-1}$. In the same region, a well-defined $-5 / 3$ energy spectra behaviour is observed over a broader wavenumber range than the fully developed region; see the inset of figure 15 in $\S 3.8$. The situation described in Valente \& Vassilicos (2012) is thus observed in the present case.

The streamwise evolution of $C_{\epsilon}$ experiences a transition between the $\operatorname{Re}_{\lambda}^{-1}$ behaviour and a region where the variations in $C_{\epsilon}$ are much smaller; see figures 12 and 13. This transition is about $x=2$. The turbulent kinetic energy budgets reported in $\S 3.10$ indicate 


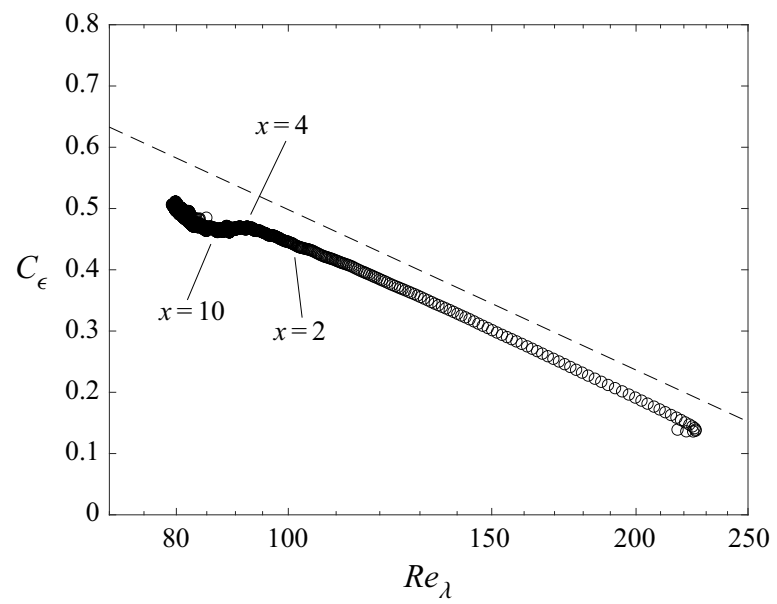

Figure 13. Plot of $C_{\epsilon}$ as a function of $R e_{\lambda}$. The dashed line follows $R e_{\lambda}^{-1}$.

that, downstream of this location, the turbulent transport terms become negligible and the mean advection of $\langle k\rangle$ equals dissipation. On the contrary, for $x<2$, this equality does not hold, and the variations of $C_{\epsilon}$ suggest a non-equilibrium condition between energy at the large scales and dissipation. It should be noted that the present results confirm the predictions reported by Tennekes \& Lumley (1972) in their (3.2.29) and (3.2.30), where transition is expected at streamwise distances from the grid that are much larger than the integral scale. In the present configuration, $x=2$ corresponds to $x \approx 6 L$.

In the theory by Richardson and Kolmogorov, the constancy of $C_{\epsilon}$ requires that turbulence is at a high Reynolds number and far from solid walls. While the small variations of $C_{\epsilon}$ in the fully developed region can be attributed to the Reynolds number, which is not very high, the steep increase in $C_{\epsilon}$ in the vicinity of the porous matrix $(x<2)$ is to be ascribed to the vicinity of the solid filaments and ultimately to non-negligible turbulent transport terms in the budget equation of $\langle k\rangle$.

\subsection{Is grid turbulence Saffman turbulence?}

In recent articles (Krogstad \& Davidson 2010; Kitamura et al. 2014) it was discussed whether grid turbulence can be considered to be of the Saffman type. Both Krogstad \& Davidson (2010) and Kitamura et al. (2014) conclude that grid turbulence is Saffman turbulence.

The theory by Saffman (1967) describes the decay of homogeneous turbulence as

$$
u_{r m s}^{2}=K C^{2 / 5} t^{-6 / 5}, \quad L=K^{\prime} C^{1 / 5} t^{2 / 5},
$$

where $K$ and $K^{\prime}$ are constants and $C$ is expressed by an invariant integral. Both equations $(3.12 a, b)$ have to hold for turbulence to be of the Saffman type. This is sometimes expressed by the requirement that $u_{r m s}^{2} L^{3}=$ const. during decay, but the latter is a necessary, not sufficient, condition for Saffman turbulence.

The exponents reported in tables 2 and 3 suggest that turbulence investigated in the present study is not of the Saffman type. Figure 14 provides graphical confirmation for this conclusion. 


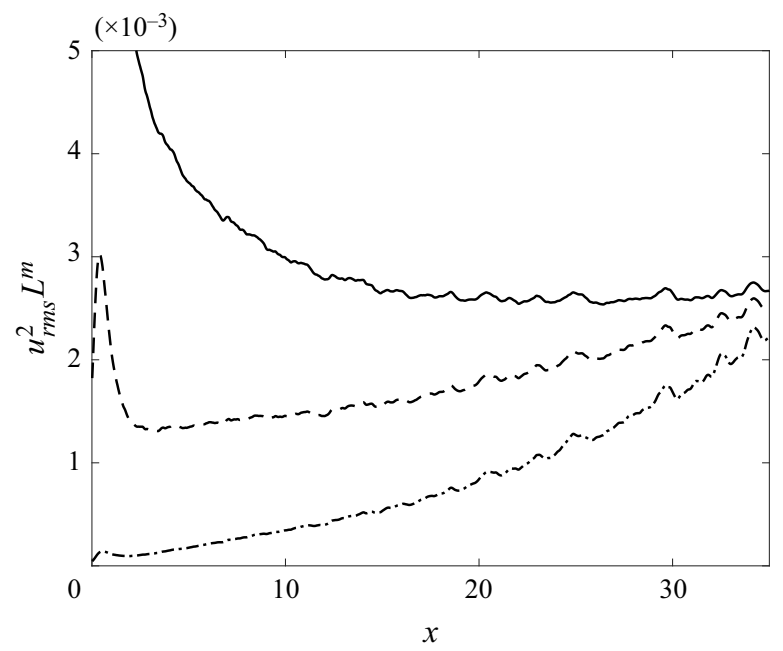

Figure 14. Streamwise evolution of the product of powers of the integral scale times powers of velocity fluctuations: solid line, $u_{r m s}^{2} L^{2}$; dashed line, $u_{r m s}^{2} L^{3}$; and dot-dashed line, $u_{r m s}^{2} L^{5}$. Saffman turbulence requires the constancy of $u_{r m s}^{2} L^{3}=$ const., not observed here.

In the fully developed region of grid turbulence, as well as in the case investigated here, the advection of turbulent kinetic energy is almost perfectly balanced by dissipation:

$$
\langle\epsilon\rangle \sim x^{-\left(n_{k}+1\right)}
$$

(see $\S 3.4$ ). Setting $n_{k}=n_{u}=n$, (3.13) combined with the definition of $C_{\epsilon}$ in (3.10) and the hypotheses $L \sim x^{q}$ and $C_{\epsilon} \sim x^{f}$ leads to the following relation:

$$
q=1-\frac{n}{2}+f
$$

As is apparent from $(3.12 a, b)$ and (3.14), grid turbulence generated at $n=6 / 5$ is not of the Saffman type unless $C_{\epsilon}$ stays constant during kinetic energy decay $(f=0)$.

\subsection{Spectral scaling and energy transfer}

One-dimensional spectra $E_{i i}\left(\kappa_{j}\right)$ are obtained from the discrete Fourier transform of the two-point velocity correlation functions along $\boldsymbol{e}_{j}$, where $\kappa_{j}$ is the $j$ th component of the wavenumber vector. Only $j=2$ and $j=3$ are used here because the streamwise direction is not homogeneous. Depending upon the pairing between velocity and wavenumber components, three distinct spectra can be calculated: streamwise spectrum $E_{s}=E_{11}\left(\kappa_{j}\right)$, longitudinal spectrum $E_{g}=E_{j j}\left(\kappa_{j}\right)$ and transverse spectrum $E_{t}=E_{i i}\left(\kappa_{j}\right)$, where $i=2$, $j=3$ or $i=3, j=2$.

While, in general, spectra at different stages of the decay could be expected to scale with different reference quantities, it is observed here that, as factors $\eta u_{\eta}^{2}, \lambda u_{r m s}^{2}$ and $L u_{r m s}^{2}$ evolve at very similar rates in the streamwise direction (see table 3), any of those can be used equivalently. Spectra scaled by $\lambda u_{r m s}^{2}$ and evaluated at increasing distance along the $x$-axis are displayed in figure 15 .

Figure 16 compares the three types of $\operatorname{spectra}\left(E_{s}, E_{g}\right.$ and $\left.E_{t}\right)$ at a coordinate $x=20$. For $\kappa_{2} \eta>0.2$, the streamwise and transverse spectra almost coincide, which is confirmation 


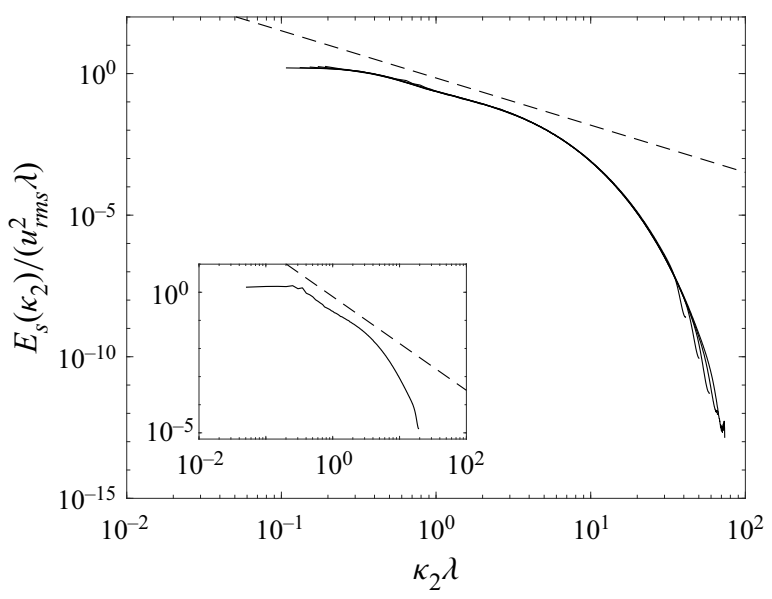

Figure 15. Streamwise one-dimensional spectra normalised in Taylor variables at increasing distance from the metal foam, $x=10,15,20,25$ and 30 . The inset displays the power-law scaling exhibited by the spectrum at $x=2$, as indicated by the dashed line with slope $-5 / 3$.

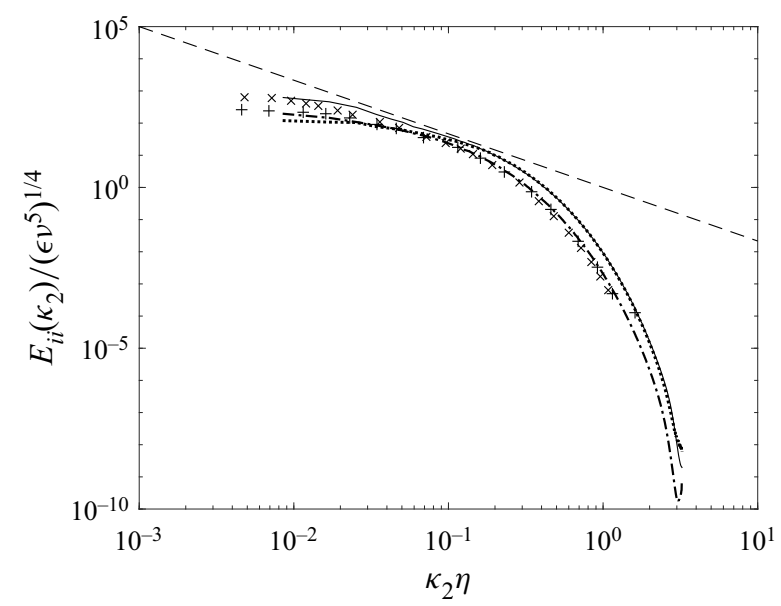

Figure 16. Comparison of streamwise, longitudinal and transverse spectra with Kolmogorov scaling at $x=$ $20\left(\operatorname{Re}_{\lambda}=81\right)$ : solid line, $E_{s}$; dot-dashed line, $E_{g}$; dotted line, $E_{t}$; and dashed line, $\left(\kappa_{2} \eta\right)^{-5 / 3}$. Symbols are longitudinal spectra of grid turbulence experiments from Comte-Bellot \& Corrsin (1971): $\times, M^{*}=50.8 \mathrm{~mm}$ $\left(x^{*} / M^{*}=98, R e_{\lambda}=65\right)$; and,$+ M^{*}=25.4 \mathrm{~mm}\left(x^{*} / M^{*}=120, \operatorname{Re}_{\lambda}=41\right)$. Asterisk ${ }^{*}$ denotes quantities expressed in dimensional form.

that anisotropy is at large scales only; see Mohamed \& Larue (1990) and Corsini \& Stalio (2020). Only a narrow range in the wavenumber space $\left(0.025<\kappa_{2} \eta<0.1\right)$ is noticed where $E_{s}$ exhibits a $-5 / 3$ behaviour. Figure 16 includes the longitudinal spectra $E_{11}\left(\kappa_{1}\right)$ of Comte-Bellot \& Corrsin (1971) for two regular grids of different mesh size and $R e_{\lambda}$ values in the same range as the present case $\left(R e_{\lambda}=65\right.$ and 41 , compared to the present $R e_{\lambda}=81$ ). Close agreement is observed between measurements in turbulence generated by classical grids and turbulence simulated in the high-porosity metal foam for $\kappa_{2} \eta>0.1$.

The present results match canonical grid turbulence spectra for $\kappa_{2} \eta>0.1$, the $-5 / 3$ law is not observed for wavenumbers $\kappa_{2} \eta>0.1$, and local isotropy is observed for $\kappa_{2} \eta>$ 0.2 . That is the range of scales where dissipation becomes non-negligible. The distinction 


\section{R. Corsini, A. Fregni, M. Spinolo and E. Stalio}

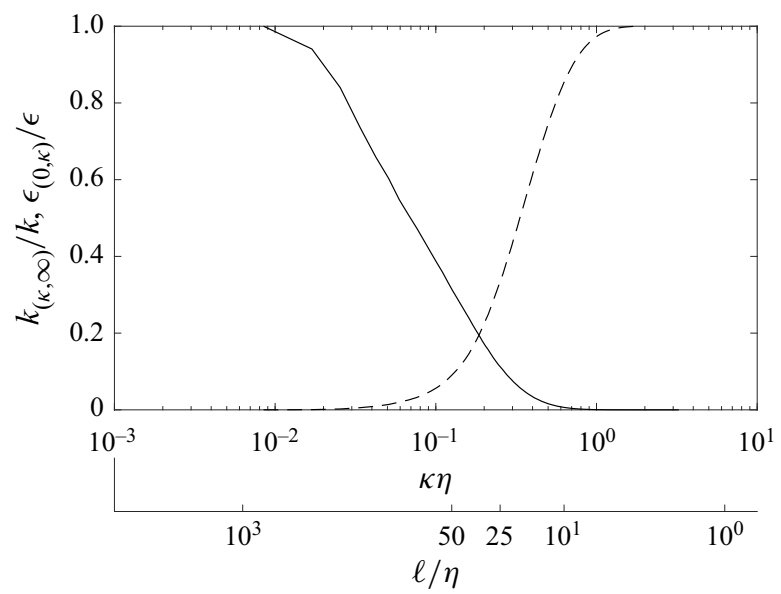

Figure 17. Normalised complement of the cumulative kinetic energy and normalised cumulative dissipation against wavenumber $\kappa \eta$ and wavelength $\ell / \eta$ at $x=20$ : solid line, $k_{(\kappa, \infty)} / k$; and dashed line, $\langle\epsilon\rangle_{(0, \kappa)} /\langle\epsilon\rangle$.

between scales containing the bulk of the energy from those responsible for dissipation is done by considering the energy spectrum $E(\kappa)$ and the dissipative spectrum $D(\kappa)=$ $2 v \kappa^{2} E(\kappa)$.

The turbulence spectrum $E(\kappa)$ is obtained by

$$
E(\kappa)=-\kappa \frac{\mathrm{d}}{\mathrm{d} \kappa} \frac{1}{2} E_{i i}(\kappa),
$$

where the summation convention is applied. The spectrum in (3.15) removes the directional information from both the velocities and the Fourier modes, as $E(\kappa)$ is given as a function of the wavenumber magnitude $\kappa=|\kappa|$.

The kinetic energy cumulated at wavenumbers lower than $\kappa$ is

$$
k_{(0, \kappa)}=\int_{0}^{\kappa} E\left(\kappa^{\prime}\right) \mathrm{d} \kappa^{\prime}
$$

the complement to $k_{(0, \kappa)}$ is indicated by $k_{(\kappa, \infty)}$. The corresponding quantities for the dissipation are obtained in the same fashion using $D(\kappa)$ and are indicated by $\langle\epsilon\rangle_{(0, \kappa)}$ and $\langle\epsilon\rangle_{(\kappa, \infty)}$. In figure 17 the fraction of cumulative turbulent kinetic energy at wavenumbers higher than $\kappa$ and the fraction of cumulative dissipation at wavenumbers lower than $\kappa$ at a distance from the foam $x=20$ are depicted as functions of $\kappa \eta$ and of the corresponding wavelength $\ell / \eta=2 \pi / \kappa \eta$. The peak of the energy spectrum occurs at $\kappa \eta \approx 0.02$ (see figure 16 ) but it may be observed that the main fraction of the kinetic energy $\left(k_{(\kappa, \infty)}=0.1 k\right)$ is contained in the range of wavenumbers up to $\kappa \eta \approx 0.25$, one decade further. The peak of dissipation is roughly at $\kappa \eta \approx 0.25$ (which corresponds to the maximum derivative in $\left.\langle\epsilon\rangle_{(0, \kappa)} /\langle\epsilon\rangle\right)$. The contribution to dissipation reaches $\langle\epsilon\rangle_{(0, \kappa)}=$ $0.9\langle\epsilon\rangle$ at $\kappa \eta \approx 0.7$. The bulk of turbulent kinetic energy is contained in motions of length scales $\ell>25 \eta \approx \frac{1}{2} L$; this range could be viewed as the energy-containing range. On the other hand, the dissipation is effective at the length scales $10 \eta<\ell<50 \eta$. The overlap between $k_{(\kappa, \infty)}$ and $\langle\epsilon\rangle_{(0, \kappa)}$ reveals that energy starts to be dissipated at a length scale where the energy content is non-negligible. 


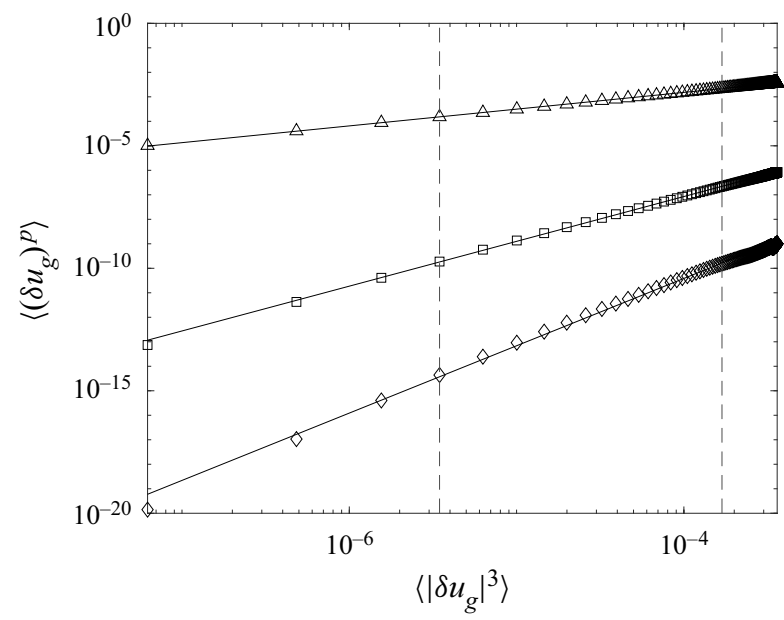

Figure 18. Log-log plot of second-, sixth- and tenth-order longitudinal structure functions versus $\left\langle\left|\delta u_{g}\right|^{3}\right\rangle$ at the position $x=25: \Delta, p=2 ; \square, p=6$; and $\diamond, p=10$. The straight lines superimposed onto the curves are the power laws resulting from the fitting procedure in the extended inertial range indicated by the dashed vertical lines. This range starts at $3 \eta$ and ends at $30 \eta$.

\subsection{Structure functions}

In this section, the local structure of turbulence is investigated at $x=25$ by analysing the scaling properties of the structure functions with separation $r$. The general definition is given by

$$
\left\langle\left[\delta u_{i, j}(\boldsymbol{x}, r)\right]^{p}\right\rangle=\left\langle\left[u_{i}\left(\boldsymbol{x}+\boldsymbol{e}_{j} r, t\right)-u_{i}(\boldsymbol{x}, t)\right]^{p}\right\rangle .
$$

Because of turbulence decay along $x$, two different structure functions are identified in this work: longitudinal structure functions $\left\langle\left(\delta u_{g}\right)^{p}\right\rangle=\left\langle\left(\delta u_{j, j}\right)^{p}\right\rangle$ and transverse structure functions $\left\langle\left(\delta u_{t}\right)^{p}\right\rangle=\left\langle\left(\delta u_{i, j}\right)^{p}\right\rangle$, where $i=2, j=3$ or $i=3, j=2$.

According to the first, original theory by Kolmogorov (1941), for high Reynolds numbers when the separation lies in the inertial subrange $\eta \ll r \ll L$, the moments of velocity difference $\left\langle\left(\delta u_{g}\right)^{p}\right\rangle$ take a universal form that depends only on $\langle\epsilon\rangle$ through the following scaling property:

$$
\left\langle\left(\delta u_{g}\right)^{p}\right\rangle \sim(\langle\epsilon\rangle r)^{\zeta_{g}^{p}} \quad \text { with } \zeta_{g}^{p}=p / 3 .
$$

In the refined similarity theory (Kolmogorov 1962), the intermittency effects are taken into account by rewriting expression (3.18) in terms of $\epsilon_{r}$, the dissipation averaged over a volume of linear dimension $r$, and assuming that $\epsilon_{r}$ has a $\log$-normal distribution,

$$
\left\langle\left(\delta u_{g}\right)^{p}\right\rangle \sim\left\langle\epsilon_{r}^{p / 3}\right\rangle r^{p / 3} \sim r^{\zeta_{g}^{p}} \quad \text { with } \zeta_{g}^{p}=\frac{1}{3} p-\frac{1}{18} \mu p(p-3),
$$

where $\mu$ is the exponent of the dissipation autocorrelation function,

$$
\langle\epsilon(\boldsymbol{x}+\boldsymbol{r}) \epsilon(\boldsymbol{x})\rangle \sim\left(\frac{L}{r}\right)^{\mu},
$$

again for inertial range separations (Monin \& Yaglom 1975).

Given the Reynolds number $R e_{\lambda} \approx 80$ of the present case, the study is conducted using the extended self-similarity (ESS) observation by Benzi et al. (1993). The $p$ th moments 


\begin{tabular}{|c|c|c|c|c|c|c|}
\hline & \multicolumn{2}{|c|}{$\begin{array}{l}\text { Present work } \\
\qquad \operatorname{Re}_{\lambda} \approx 80\end{array}$} & \multirow{2}{*}{$\begin{array}{c}\text { Camussi } \\
R e_{\lambda} \approx 37 \\
\text { ESS }\end{array}$} & \multirow{2}{*}{$\begin{array}{c}\text { Zhou } \\
R e_{\lambda}=75 \\
\text { ESS }\end{array}$} & \multirow{2}{*}{$\begin{array}{c}\text { Boratav } \\
R e_{\lambda} \sim 100 \\
\text { ESS }\end{array}$} & \multirow{2}{*}{$\begin{array}{c}\text { Iyer } \\
R e_{\lambda}=1300 \\
\text { Direct }\end{array}$} \\
\hline & Direct & ESS & & & & \\
\hline$p=2$ & $0.722 \pm 0.027$ & $0.684 \pm 0.005$ & $0.68 \pm 0.006$ & 0.70 & 0.6931 & $0.72 \pm 0.002$ \\
\hline$p=4$ & $1.343 \pm 0.052$ & $1.296 \pm 0.008$ & $1.29 \pm 0.008$ & 1.32 & 1.2818 & $1.30 \pm 0.005$ \\
\hline$p=6$ & $1.852 \pm 0.076$ & $1.829 \pm 0.032$ & $1.81 \pm 0.029$ & 1.86 & 1.7804 & $1.78 \pm 0.010$ \\
\hline$p=8$ & $2.241 \pm 0.128$ & $2.303 \pm 0.068$ & - & 2.34 & 2.2123 & $2.18 \pm 0.021$ \\
\hline$p=10$ & $2.507 \pm 0.272$ & $2.748 \pm 0.126$ & 一 & - & 2.6000 & - \\
\hline$p=12$ & $2.679 \pm 0.509$ & $3.187 \pm 0.208$ & - & 一 & 2.9614 & - \\
\hline
\end{tabular}

Table 4. Scaling exponents for the longitudinal structure functions of even orders up to $p=12$ calculated using the direct and the ESS methods. Comparison of the present work with the results from Camussi et al. (1996), Zhou \& Antonia (2000), Boratav \& Pelz (1997) and Iyer et al. (2017).

of velocity differences $\left\langle\left(\delta u_{g}\right)^{p}\right\rangle$ calculated at moderate Reynolds number are characterised by the same scaling exponents as in the high-Reynolds-number case when $\left\langle\left(\delta u_{g}\right)^{p}\right\rangle$ are treated as functions of $\left\langle\left|\delta u_{g}\right|^{3}\right\rangle$. The resulting exponents are indicated by $\xi_{g}^{p}=\zeta_{g}^{p} / \zeta_{g}^{3}$ to discriminate them from those evaluated directly, $\zeta_{g}^{p}$. The quality of the ESS scaling is displayed in figure 18 and in table 4 . Errors are calculated by summing the uncertainty obtained from the estimate of scaling in the two spatial directions $y$ and $z$ and by modifying the scaling range over which $\xi_{g}^{p}$ is evaluated in the interval $[3,30] \eta$. Data from the literature reported for comparison in table 4 are at $R e_{\lambda}$ comparable to the present, i.e. of order $\sim 10^{2}$, except for those by Iyer, Sreenivasan \& Yeung (2017), which are obtained at $R e_{\lambda} \approx 1300$ and a high degree of isotropy.

Figure 19(a) shows scaling exponents $\xi_{g}^{p}$ and $\xi_{t}^{p}$ computed using the ESS method, compared to the original predictions by Kolmogorov (1941, KM41) extended to $p>3$, the refined similarity hypotheses of Kolmogorov (1962, KM62), the intermittency $\beta$ model by Frisch, Sulem \& Nelkin (1978) and the model by She \& Leveque (1994). The transverse scaling exponents $\xi_{t}^{p}$ in figure 19 are obtained by making $\left\langle\left(\delta u_{t}\right)^{p}\right\rangle$ depend on the magnitude of transverse velocity differences for $p=3$ rather than the longitudinal, thus following the ESS method (Camussi et al. 1996; Grossmann, Lohse \& Reeh 1997). The refined similarity hypotheses are found to hold, but there is a visible tendency of $\xi_{t}^{p}$ to deviate from the longitudinal scaling for $p \geqslant 4$. This is to be ascribed to the Reynolds number and the residual anisotropy of the flow, as argued by Iyer et al. (2017).

Figure 19(b) displays the evolution of the intermittency exponent $\mu$ along the streamwise direction; the quantity defined in (3.20) is also employed in the $\beta$-model by Frisch et al. (1978). The $\mu$ exponent is calculated directly from the slope of the correlation coefficient obtained by dividing the autocorrelation of dissipation by $\langle\epsilon\rangle^{2}$ for separations in the inertial range; see figure 20. A second possible way to obtain $\mu$ is by using the expression relating the longitudinal sixth-order moment to the autocorrelation of dissipation (Frisch et al. 1978):

$$
\frac{\left\langle\left(\delta u_{g}\right)^{6}\right\rangle}{r^{2}} \sim\langle\epsilon(\boldsymbol{x}+\boldsymbol{r}) \epsilon(\boldsymbol{x})\rangle .
$$

The two quantities are reported in figure $19(b)$. As the reference value reported in many studies for the intermittency exponent is $\mu=0.25 \pm 0.05$, which is deemed to be valid for high-Reynolds-number flows (Pope 2000), the values calculated here suggest 

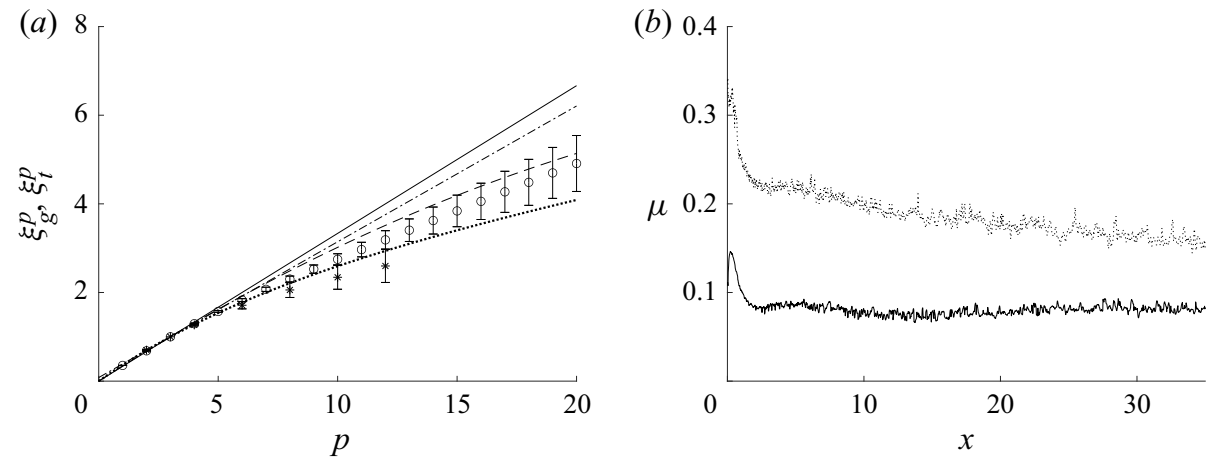

Figure 19. (a) Scaling exponents $\xi_{g}^{p}$ and $\xi_{t}^{p}$ computed with the ESS method versus $p$ for orders up to 20 $(x=25)$. In the same plot there are the results from different intermittency models assuming $\mu=0.08: \circ$, $\xi_{g}^{p} ; *, \xi_{t}^{p}$; solid line, KM41 model; dashed line, KM62 model; dot-dashed line, $\beta$ model; and dotted line, She-Leveque model. $(b)$ Behaviours of the intermittency exponent along $x$ computed according to two different methods: solid line, based on $\langle\epsilon(\boldsymbol{x}+\boldsymbol{r}) \epsilon(\boldsymbol{x})\rangle /\langle\epsilon\rangle^{2} \sim(L / r)^{\mu}$; and dotted line, $\mu=2-\xi_{g}^{6}$.

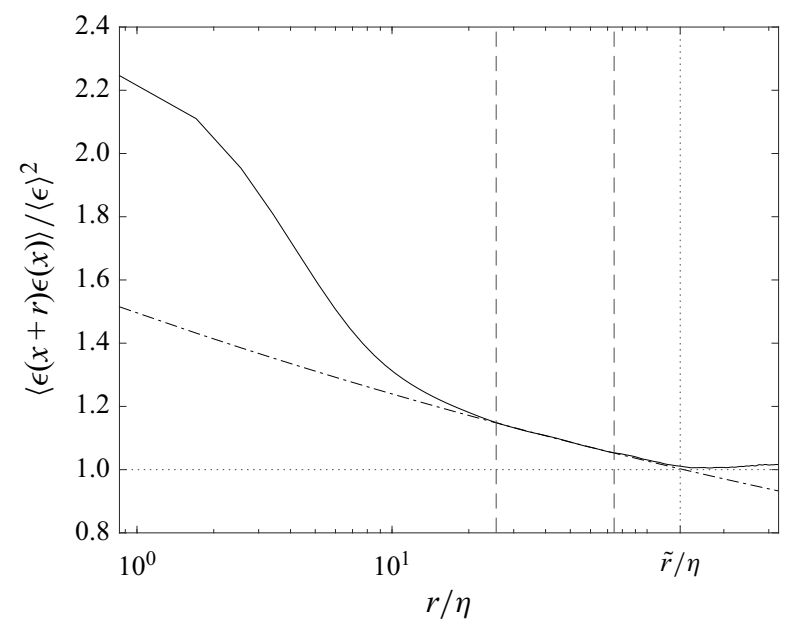

Figure 20. Dissipation correlation coefficient at $x=25$ : solid line, $\langle\epsilon(\boldsymbol{x}+\boldsymbol{r}) \epsilon(\boldsymbol{x})\rangle /\langle\epsilon\rangle^{2}$ as calculated from its definition; and dot-dashed line, $a_{d}(r / \eta)^{d}$, where $a_{d}=1.07$ and $d=-0.082$. The dashed lines delimit the interval used for the power-law fitting procedure, $25 \eta<r<75 \eta$, and the dotted lines demarcate the decorrelation scale $\tilde{r}$.

that the effect generated by intermittent structures on small-scale turbulence in the moderate-Reynolds-number range is less intense than at high Reynolds numbers. It is noticed that the method based on sixth-order structure functions, (3.21), displays a decay in the $x$-direction that is not expected nor recovered in the calculation of $\mu$ directly from the definition (3.20).

The decorrelation scale $\tilde{r}$ is defined as the length of decorrelation of the instantaneous dissipation $\epsilon$, i.e. the separation scale $r$ where $\langle\epsilon(\boldsymbol{x}+\boldsymbol{r}) \epsilon(\boldsymbol{x})\rangle /\langle\epsilon\rangle^{2}$ becomes unitary with a $1 \%$ error. In figure 21 the development of $\tilde{r}$ along $x$ is compared to that of the integral scale $L$. The decorrelation scale can be considered as a very large length scale, which 


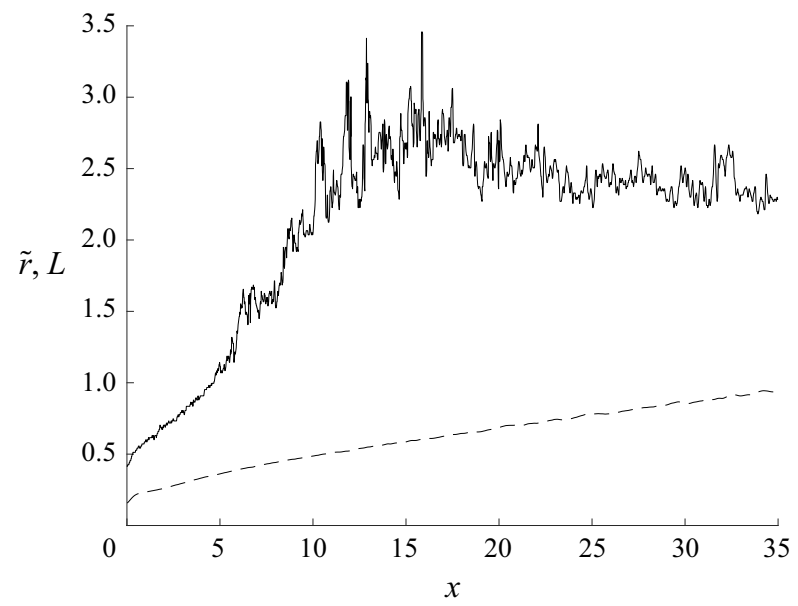

Figure 21. Streamwise evolution of the decorrelation scale in comparison with the integral scale: solid line, $\tilde{r}$; and dashed line, $L$. For $x=25, \tilde{r} / \eta=135$ and $L / \eta=45$.

in homogeneous isotropic turbulence depends on the Reynolds number and also the intermittency characteristics of the flow.

\subsection{Turbulent energy budgets}

The equation governing the transport of $\langle k\rangle$ in a statistically steady case can be written in symbols as

$$
-\mathcal{A}=\mathcal{T}_{p}+\mathcal{T}_{t}+\mathcal{D}_{v}+\mathcal{P}-\langle\tilde{\epsilon}\rangle
$$

where $\mathcal{A}$ is the contribution by mean advection and $\mathcal{T}_{p}, \mathcal{T}_{t}$ and $\mathcal{D}_{v}$ represent pressure, turbulent and diffusive transports; $\mathcal{P}$ and $\langle\tilde{\epsilon}\rangle$ stand for the production and pseudo-dissipation rate of turbulent kinetic energy, defined as

$$
\langle\tilde{\epsilon}\rangle=\frac{1}{\operatorname{Re}_{d_{p}}}\left\langle\frac{\partial u_{i}}{\partial x_{j}} \frac{\partial u_{i}}{\partial x_{j}}\right\rangle .
$$

As the difference $\langle\epsilon\rangle-\langle\tilde{\epsilon}\rangle$ is seldom important (Pope 2000), in this section $\langle\epsilon\rangle$ is used.

In grid turbulence cases, where mean velocity gradients are zero and cross-flow directions $y$ and $z$ are homogeneous, the production term vanishes:

$$
\mathcal{P}=-\left\langle u_{i} u_{j}\right\rangle \frac{\partial\left\langle U_{i}\right\rangle}{\partial x_{j}}=0 .
$$

In addition, for the Reynolds number computed here, the molecular contribution to transport is negligible. The resulting expression for the budget equation (3.22) thus becomes

$$
\underbrace{\langle U\rangle \frac{\mathrm{d}\langle k\rangle}{\mathrm{d} x}}_{-\mathcal{A}}=\underbrace{-\frac{\mathrm{d}\langle u p\rangle}{\mathrm{d} x}}_{\mathcal{T}_{p}} \underbrace{-\frac{\mathrm{d}\langle u k\rangle}{\mathrm{d} x}}_{\mathcal{T}_{t}}-\langle\tilde{\epsilon}\rangle,
$$

where the single terms are distributed along the $x$-axis as depicted in figure 22 .

Two regions can be identified in figure 22: a near-field region where the three conservative terms redistribute the kinetic energy along $x$, and a far-field region where 

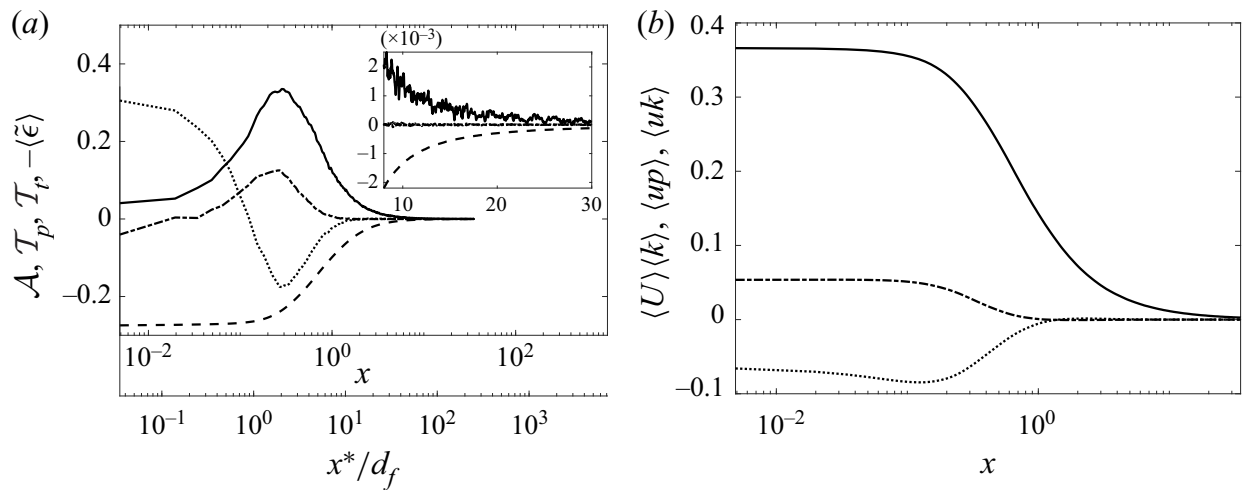

Figure 22. Turbulent kinetic energy along the streamwise direction in logarithmic plots. In panel $(a)$ budget terms are reported: solid line, $\mathcal{A}$; dot-dashed line, $\mathcal{T}_{p}$; dotted line, $\mathcal{T}_{t}$; and dashed line, $-\langle\tilde{\epsilon}\rangle$. Panel $(b)$ presents turbulent kinetic energy fluxes: solid line, $\langle U\rangle\langle k\rangle$; dot-dashed line, $\langle u p\rangle$; and dotted line, $\langle u k\rangle$. The inset in $(a)$ displays an enlargement of the budget terms in the range $I_{d}$ in linear scale.

pressure and turbulent transports become negligible. Thus dissipation is solely balanced by turbulent kinetic energy advection; see figure 22( $a$ ) and its inset. A possible downstream boundary for the near-field region can be set at $x=2$, where the turbulent and pressure transports become 100 times smaller than advection and dissipation.

The near-field region can be subdivided into two subregions. By considering the ligament diameter $d_{f}$ as the length scale, a region very close to the metal foam is identified for $x^{*} / d_{f}<0.5$, where turbulent transport is larger than the advective one. This region is characterised by the sustainment of turbulence by means of streamwise fluctuations, which is counter-balanced by dissipation, as pressure and advective transports account for negligible shares. The second subregion extends for $0.5<x^{*} / d_{f}<14$, where turbulent kinetic energy is provided through pressure and advective mechanisms while dissipation and turbulent transport drain $\langle k\rangle$; see figure 22(a).

Data gathered by Norberg (1998) indicate that the vortex formation length $\ell_{f}$ for the present Reynolds number based on the mean diameter of the filaments, $R e_{d_{f}}=560$, lies in the range $1.5 d_{f}<\ell_{f}<2 d_{f}$; see Bloor, Gerrard \& Lighthill (1966) for other possible definitions of the vortex formation length. Figure 22(a) displays that this range matches the streamwise location where all the transport terms in (3.25) peak. Therefore, the present data suggest that kinetic energy is originated in the second part of the near-field region, where transport mechanisms show local peaks. Turbulent kinetic energy $\langle k\rangle$ is drained from here by the turbulent transport, which transfers turbulent fluctuations upstream, as indicated by the negative sign of the turbulent flux (see figure $22 b$ ), feeding the region very close to the metal foam. On the other hand, pressure and advective mechanisms are found to provide turbulent kinetic energy in the entire region $0.5<x^{*} / d_{f}<14$, and the signs of the relative fluxes indicate that velocity fluctuations are transferred downstream. At the coordinate $x^{*} / d_{f}=14(x=2)$, fluxes $\langle u p\rangle$ and $\langle u k\rangle$ become constant in the streamwise direction, leading to the budget reported in (3.4) for $x>2$, which is typical in developed decaying flows; see figure $22(a)$ and its inset, which reports the budget terms in the $I_{d}$ region.

In order to separately assess the behaviour of streamwise and cross-flow velocity fluctuations, the transport equations for velocity variances are analysed. The budget of 


\section{R. Corsini, A. Fregni, M. Spinolo and E. Stalio}

streamwise velocity variance reads

$$
\underbrace{\langle U\rangle \frac{\mathrm{d}\left\langle u^{2}\right\rangle}{\mathrm{d} x}}_{-\mathcal{A}^{u}}=\underbrace{-\frac{\mathrm{d}\left\langle u^{3}\right\rangle}{\mathrm{d} x}}_{\mathcal{T}_{t}^{u}} \underbrace{-2 \frac{\mathrm{d}\langle u p\rangle}{\mathrm{d} x}}_{\mathcal{T}_{p}^{u}} \underbrace{+2\left\langle p \frac{\partial u}{\partial x}\right\rangle}_{\mathcal{S}^{u}}-\left\langle\widetilde{\epsilon}_{u}\right\rangle,
$$

where, from left to right, the first three terms, respectively, represent the advective, turbulent and pressure transports of $\left\langle u^{2}\right\rangle,\langle p \partial u / \partial x\rangle$ is the pressure strain term and $\widetilde{\epsilon_{u}}$ stands for the $u$-variance pseudo-dissipation rate, $\tilde{\epsilon_{u}}=2\left(\left(\partial u / \partial x_{j}\right)\left(\partial u / \partial x_{j}\right)\right) / \operatorname{Re}_{d_{p}}$. The diffusive transport is again neglected with respect to the other terms. Equations similar to (3.26) can be derived for the transverse velocity components, $v$ and $w$. As the flow is isotropic on $y-z$ planes, statistics for the transverse velocity component are obtained by averaging results along $y$ and $z$. For conciseness, the transverse velocity component is indicated by $v$ and its direction by $y$,

$$
\underbrace{\langle U\rangle \frac{\mathrm{d}\left\langle v^{2}\right\rangle}{\mathrm{d} x}}_{-\mathcal{A}^{v}}=\underbrace{-\frac{\mathrm{d}\left\langle u v^{2}\right\rangle}{\mathrm{d} x}}_{\mathcal{T}_{t}^{v}} \underbrace{2\left\langle p \frac{\partial v}{\partial y}\right\rangle}_{\mathcal{S}^{v}}-\left\langle\widetilde{\epsilon}_{v}\right\rangle,
$$

where the terms have the same interpretation as in (3.26), and the pseudo-dissipation rate of the $v$-variance is computed as $\widetilde{\epsilon_{v}}=2\left(\left(\partial v / \partial x_{j}\right)\left(\partial v / \partial x_{j}\right)\right) / R e_{d_{p}}$. Note that, with respect to equation (3.25), budgets of velocity component variances include the pressure strain terms. As will be shown in the following, the role of these terms is to redistribute kinetic energy among the velocity components and thus it is responsible for the 'return to isotropy' in homogeneous turbulence. Such a phenomenon has been studied for several decades as it is involved in second-order turbulence models; see, for example, the works by Rotta (1951) and Lumley \& Newman (1977). Pressure strain terms are not included in (3.25) as their sum $\mathcal{S}^{u}+2 \mathcal{S}^{v}$ vanishes because of incompressibility.

Figures 23 and 24 show the velocity variance budgets and fluxes along the streamwise coordinate. It appears that transport mechanisms are more intense in the $\left\langle u^{2}\right\rangle$ budget with respect to $\left\langle v^{2}\right\rangle$, while dissipations $\left\langle\tilde{\epsilon}_{u}\right\rangle$ and $\left\langle\tilde{\epsilon}_{v}\right\rangle$ are almost equal. The behaviour of the pressure strain terms reveals that turbulent energy is drained from the streamwise velocity fluctuations, as $\mathcal{S}^{u}$ represents a sink term in the $\left\langle u^{2}\right\rangle$ budget, and provided to cross-flow velocity fluctuations, where $\mathcal{S}^{v}$ acts as a source; see figures 23(a) and 24(a). The role of $\mathcal{S}^{u}$ and $\mathcal{S}^{v}$ does not change along the whole streamwise extension of the domain, as shown by the insets in figures 23(a) and 24(a). This occurs because, as reported in $\$ 3.2$, flow anisotropy is conserved in the flow domain considered. In the decaying region $I_{d}$, the pressure strain terms maintain an intensity comparable to the advective and dissipation terms, suggesting that the isotropic condition would have been reached in a longer computational domain.

The profiles in figures 23 and 24 show that the $u$-variance terms behave very similarly to the budgets and fluxes of turbulent kinetic energy reported in figure 22. In particular, the peaks of turbulent, advective and pressure transports are located at the same distance from the metal foam, comparable to the vortex formation length. This suggests that velocity fluctuations are more intensely triggered along the streamwise direction with respect to the transverse ones. On the other hand, the $v$-variance terms peak slightly downstream with respect to terms in the $\left\langle u^{2}\right\rangle$ and $\langle k\rangle$ budgets, and the negative peak of turbulent transport is not very intense (see figure $24 a$ ), but it drains enough energy to sustain cross-flow fluctuations in the region just downstream of the metal foam. Another difference with respect to the $\left\langle u^{2}\right\rangle$ terms is the positive - yet not that intense - turbulent flux very close 

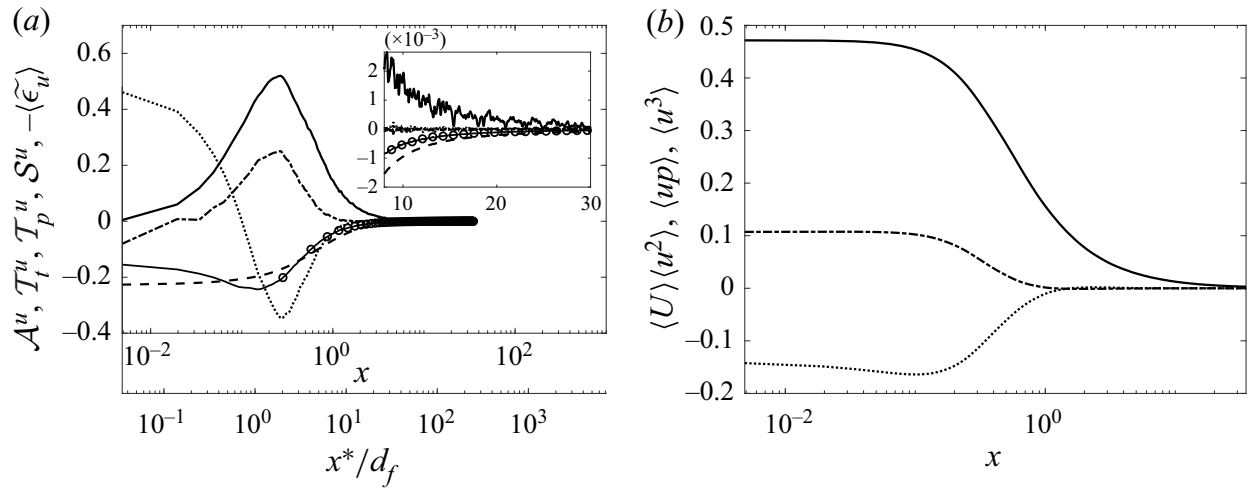

Figure 23. Variance of streamwise velocity component along the streamwise direction in logarithmic plots. In panel ( $a$ ) budget terms are reported: solid line, $\mathcal{A}^{u}$; dotted line, $\mathcal{T}_{t}^{u} ;$ dot-dashed line, $\mathcal{T}_{p}^{u}$; solid line with circles, $\mathcal{S}^{u}$; and dashed line, $-\left\langle\tilde{\epsilon}_{u}\right\rangle$. Panel $(b)$ presents $\left\langle u^{2}\right\rangle$ fluxes: solid line, $\langle U\rangle\left\langle u^{2}\right\rangle$; dot-dashed line, $\langle u p\rangle$; and dotted line, $\left\langle u^{3}\right\rangle$. The inset in $(a)$ displays an enlargement of the budget terms in the range $I_{d}$ in linear scale.
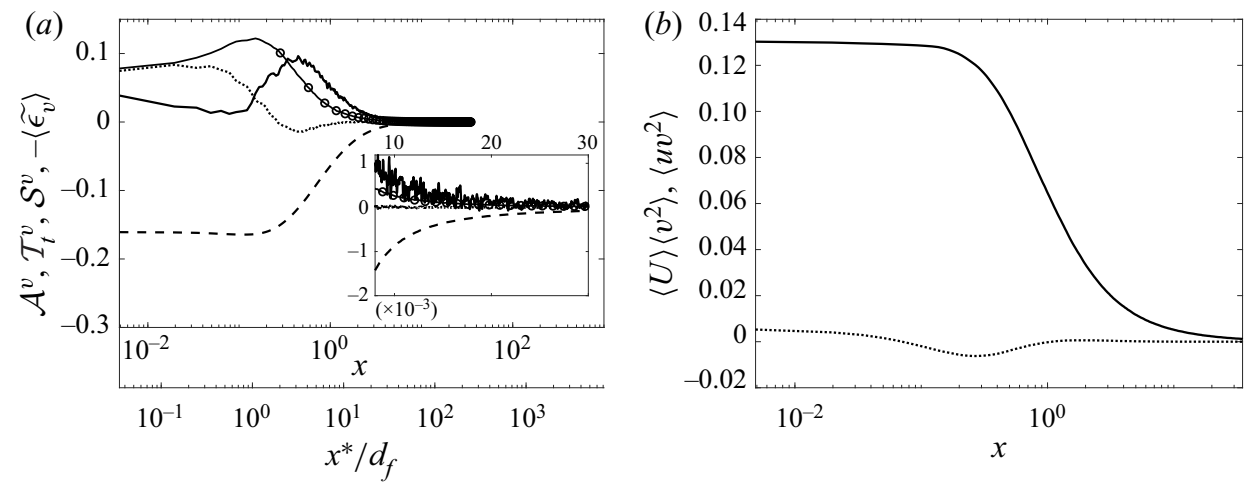

Figure 24. Variance of cross-flow velocity component along the streamwise direction in logarithmic plots. In panel (a) budget terms are reported: solid line, $\mathcal{A}^{v}$; dotted line, $\mathcal{T}_{t}{ }^{v}$; solid line with circles, $\mathcal{S}^{v}$; and dashed line, $-\left\langle\tilde{\epsilon_{v}}\right\rangle$. Panel $(b)$ presents $\left\langle v^{2}\right\rangle$ fluxes: solid line, $\langle U\rangle\left\langle v^{2}\right\rangle$; and dotted line, $\left\langle u v^{2}\right\rangle$. The inset in $(a)$ displays an enlargement of the budget terms in the range $I_{d}$ in linear scale.

to the metal foam, indicating that cross-flow fluctuations are more correlated with positive $u$-fluctuations in this region (see figure $24 b$ ).

\section{Conclusions}

An analysis is presented of the turbulent flow behind a synthetic metal foam layer of thickness equal to five times the mean pore diameter. Unlike classical grid turbulence geometries, the metal foam ligaments are variably oriented, unevenly spaced and in general less ordered. Similar to classical grids, metal foams are mainly characterised by two length scales i.e. the pore diameter and the ligament thickness. The analysis encompasses one single Reynolds number $R e_{d_{p}}=4000$ and $\varepsilon=0.92$ but the effect of a different porosity $(\varepsilon=0.97)$ on selected quantities is addressed in the Appendix.

The analysis of the turbulent kinetic energy budget suggests that turbulence is triggered in the region close to the metal foam, approximately at a distance equal to the vortex formation length indicated by Norberg (1998), $x^{*} / d_{f} \approx 2$. In the near-field region, $x<2$, 


\section{R. Corsini, A. Fregni, M. Spinolo and E. Stalio}

turbulent kinetic energy is distributed by transport mechanisms; the turbulent transport moves $\langle k\rangle$ upstream, sustaining fluctuations in close proximity to the metal foam, while pressure and advective transports provide velocity fluctuations to larger $x$-coordinates. At $x=2$, pressure and turbulent transports are found to be negligible with respect to the other terms, entailing for $x>2$ the expected behaviour of grid turbulence, where viscous dissipation is balanced by the mean advection of $\langle k\rangle$. Budgets of streamwise and cross-flow velocity variances indicate that fluctuations along $x$ are the most intensely triggered and pressure strain terms act to redistribute turbulent energy towards the isotropic condition, which however is not observed due to the limited domain extension.

In that same near-field region, $R e_{\lambda}$ decreases steeply and the dissipation rate coefficient $C_{\epsilon}$ approximates $C_{\epsilon} \propto \operatorname{Re}_{\lambda}^{-1}$ while $L / \lambda$ remains almost constant. These observations, in conjunction with the typical $-5 / 3$ slope in the turbulent power spectra, represent a typical behaviour already observed in the literature (Valente \& Vassilicos 2012). Given that this occurs very close to the porous matrix, where the hypotheses for the Richardson cascade are not verified, a considerable variation in $C_{\epsilon}$ is not interpreted here as an anomalous behaviour.

The developed region is defined here as the region where $u_{r m s}$ decays following a power law and starts at $x_{\min }=7.98$. Besides $\langle k\rangle$, a number of relevant quantities, like the integral length scale, the Taylor microscale and the Kolmogorov scale of the flow, are observed to follow a power-law behaviour. The exponents obtained from a least-squares fit are in many cases very close to values predicted and measured in classical grid turbulence experiments. Given $n_{k}=1.14$ and $q=0.52$ (where $n_{k}$ and $q$ are the exponents of the power laws for $\langle k\rangle$ and $L$ ), the constancy of $u_{r m s}^{2} L^{3}$ does not hold in the developed region simulated here, and thus the turbulence does not follow the theory by Saffman.

Structure functions of different orders are calculated at a fixed position $x=25$ in the fully developed region. The extended self-similarity is used to calculate the scaling exponents. The results are interpreted in the light of the refined similarity hypothesis. The intermittency exponent $\mu$ computed directly from its definition is seen to behave uniformly within the computational domain. It reveals that intermittency is not very intense at the moderate Reynolds number of this study. The decorrelation length of dissipation $\tilde{r}$ is larger than the integral scale. It depends on the Reynolds number as well as the intermittency characteristics of the flow.

Acknowledgements. The authors would like to thank S. Chibbaro and A. Cimarelli for fruitful discussions and suggestions.

Funding. The authors acknowledge the CINECA award under the ISCRA initiative, for the availability of high-performance computing resources and support.

Declaration of interests. The authors report no conflict of interest.

\section{Author ORCIDs.}

(ㄱ) R. Corsini https://orcid.org/0000-0001-9342-0773;

A. Fregni https://orcid.org/0000-0002-1810-3281;

E. Stalio https://orcid.org/0000-0001-6983-4204.

\section{Appendix. A glance at the effect of porosity}

The aim of this appendix is to verify the extent to which turbulent flow statistics in the lee of an open-cell metal foam layer are affected by porosity. The results obtained for a foam of porosity $\varepsilon=0.97$ are compared here against the $\varepsilon=0.92$ case, as investigated in the main 


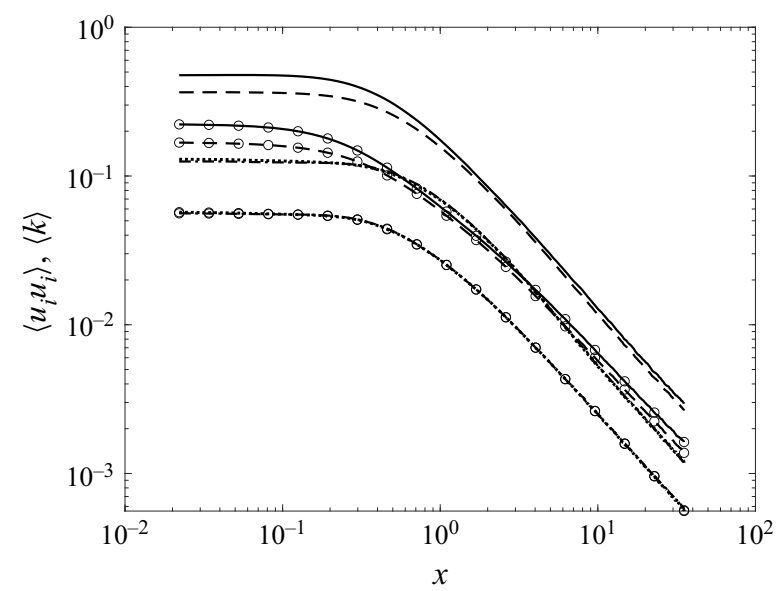

Figure 25. Streamwise evolution of velocity fluctuations and turbulent kinetic energy for the $\varepsilon=0.97$ and $\varepsilon=0.92$ cases on the coarse computational grid: solid line, $\langle u u\rangle$; dot-dashed line, $\langle v v\rangle$; dotted line, $\langle w w\rangle$; and dashed line, $\langle k\rangle$. The lines with circles mark the case with higher porosity $\varepsilon=0.97$.

body of the paper. The two foams have equal pore size but different ligament thickness, i.e. the foam with larger porosity is characterised by a thinner ligament, $d_{f}^{\prime}<d_{f}$. The comparison is conducted by means of DNS performed with the same numerical parameters as outlined in $\S 2$ except for the computational grid, which consists of $n_{x}=2049$ and $n_{y}=n_{z}=512$ grid nodes.

The porous matrix of higher porosity generates a turbulent field where fluctuations are less intense in all the spatial directions. Application of the fitting procedure outlined in $\S 3.3$ on the high-porosity foam provides decay exponents for $u_{r m s}^{2}$ and $\langle k\rangle$ of $n_{u}^{\prime}=1.05$ and $n_{k}^{\prime}=1.08$, respectively. The results obtained on the coarse grid for the $\varepsilon=0.92$ case yield $n_{u}=1.12$ and $n_{k}=1.13$; see table 2 for comparisons against results on the fine mesh. The power-law decay of the turbulent kinetic energy begins more upstream than in the $\varepsilon=0.92$ case; see figure 25 .

Inside the developed region, the Kolmogorov and the longitudinal integral length scales evaluated for $\varepsilon=0.97$ behave according to power laws with growing rates estimated by the exponents $s^{\prime}=0.54$ and $q^{\prime}=0.47$, respectively (see table 3 for notation). The lower-porosity case exhibits decay exponents along the $x$-direction of $s=0.56$ and $q=$ 0.51 (see table 3 for comparisons against results on the fine mesh) but the Kolmogorov and the integral scales are different in magnitude, as displayed by figure 26( $a$ ) and $(b)$. Associated with the smaller kinetic energy content of the flow and a smaller dissipation rate observed at $\varepsilon=0.97$, the turbulence induced by the higher-porosity foam is characterised by a larger Kolmogorov scale and a smaller integral scale.

Figure 27 displays the effects of porosity on the terms of the turbulent kinetic energy budget. The transport mechanisms of $\langle k\rangle$ for $\varepsilon=0.97$ are the same as described in $\S 3.10$ for $\varepsilon=0.92$ but are characterised by an upstream shift of the transport peaks with respect to that case. This shift is consistent with the reduction of the vortex formation length associated with a smaller ligament thickness, as indicated by Norberg (1998) and reported in $\S 3.10$.

In summary, while the results obtained for $\varepsilon=0.92$ show small quantitative differences with respect to $\varepsilon=0.97$, no significant discrepancies are observed between the two cases. An upstream shift in the peaks of $\langle k\rangle$ transports characterises the higher-porosity case, 

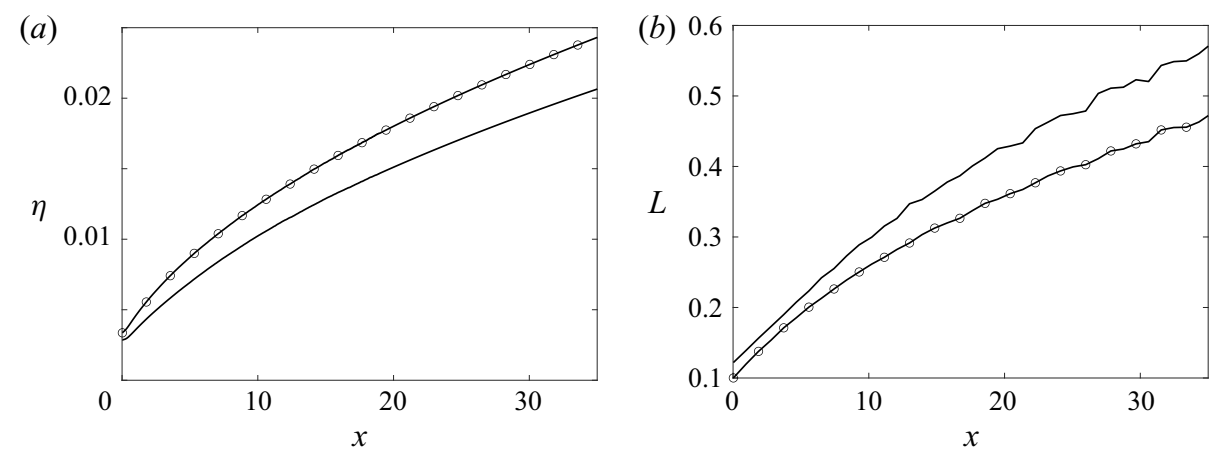

Figure 26. Comparison between the streamwise distributions of the Kolmogorov scale $(a)$ and the longitudinal integral scale $(b)$ for the $\varepsilon=0.97$ and $\varepsilon=0.92$ cases on the coarse computational grid: solid line, length scales for $\varepsilon=0.92$; and solid line with circles, length scales for $\varepsilon=0.97$.

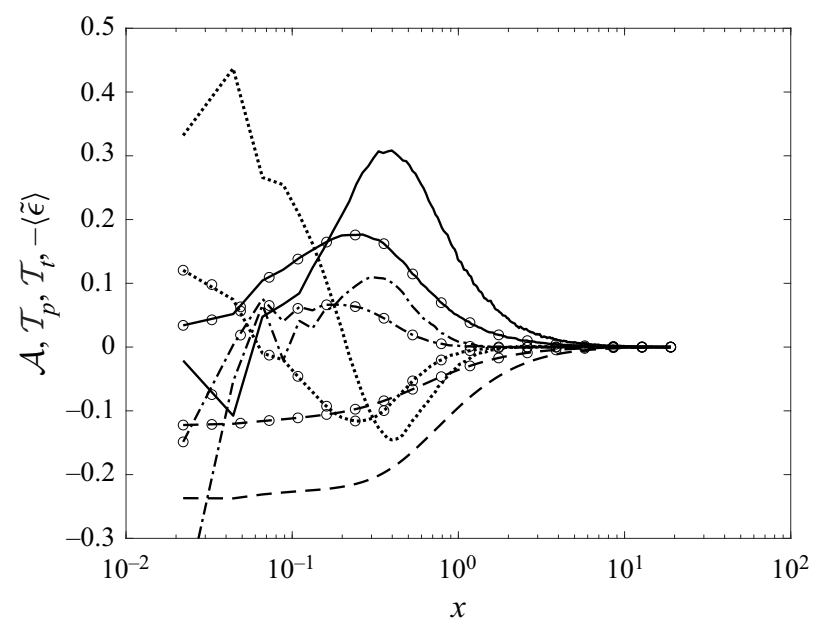

Figure 27. Comparison between the turbulent kinetic energy budget terms for the $\varepsilon=0.97$ and $\varepsilon=0.92$ cases on the coarse computational grid: solid line, $\mathcal{A}$; dot-dashed line, $\mathcal{T}_{p}$; dotted line, $\mathcal{T}_{t}$; and dashed line, $-\langle\tilde{\epsilon}\rangle$. The lines with circles mark the case with higher porosity $\varepsilon=0.97$.

which in turn implies a reduced vortex formation length and the achievement of power-law decays at shorter distances from the porous matrix. The size of such displacement is apparently very small.

\section{REFERENCES}

August, A., Ettrich, J., Rölle, M., Schmid, S., Berghoff, M., Selzer, M. \& Nestler, B. 2015 Prediction of heat conduction in open-cell foams via the diffuse interface representation of the phase-field method. Intl J. Heat Mass Transfer 84, 800-808.

BATCHELOR, G.K. 1948 Energy decay and self-preserving correlation functions in isotropic turbulence. Q. Appl. Maths 6, 97-116.

Batchelor, G.K. \& Townsend, A.A. 1948 Decay of isotropic turbulence in the initial period. Proc. R. Soc. Lond. A 193, 539-558.

Benzi, R., Ciliberto, S., Tripiccione, R., Baudet, C., Massaioli, F. \& Succi, S. 1993 Extended self-similarity in turbulent flows. Phys. Rev. E 48, R29-R32. 


\section{Turbulent flow past a metal foam}

Bloor, M.S., Gerrard, J.H. \& Lighthill, M.J. 1966 Measurements on turbulent vortices in a cylinder wake. Proc. R. Soc. Lond. A 294 (1438), 319-342.

Boomsma, K., Poulikakos, D. \& Ventikos, Y. 2003 Simulations of flow through open cell metal foams using an idealized periodic cell structure. Intl J. Heat Fluid Flow 24, 825-834.

BoratAV, O.N. \& PELZ, R.B. 1997 Structures and structure functions in the inertial range of turbulence. Phys. Fluids 9, 1400-1415.

Calmidi, V.V. \& Mahajan, R.L. 2000 Forced convection in high porosity metal foams. Trans. ASME J. Heat Transfer 122, 557-565.

Camussi, R., Barbagallo, D., Guj, G. \& Stella, F. 1996 Transverse and longitudinal scaling laws in non-homogeneous low Re turbulence. Phys. Fluids 8, 1181-1191.

Comte-Bellot, G. \& CORRSin, S. 1966 The use of a contraction to improve the isotropy of grid-generated turbulence. J. Fluid Mech. 25, 657-682.

Comte-Bellot, G. \& CORRSin, S. 1971 Simple Eulerian time correlation of full- and narrow-band velocity signals in grid-generated, 'isotropic' turbulence. J. Fluid Mech. 48, 273-337.

Corsini, R. \& STAlio, E. 2020 Direct numerical simulation of turbulence in the wake of a metal foam. Intl Commun. Heat Mass Transfer 115, 104599.

Frisch, U., Sulem, P. \& NELKIN, M. 1978 A simple dynamical model of intermittent fully developed turbulence. J. Fluid Mech. 87, 43-164.

Gautier, R., LAizet, S. \& LAmballais, E. 2014 A DNS study of jet control with microjets using an immersed boundary method. Intl J. Comput. Fluid Dyn. 28, 393-410.

GeORge, W.K. 1992 The decay of homogeneous isotropic turbulence. Phys. Fluids A 4, 1492-1509.

Gomes-Fernandes, R., Ganapathisubramani, B. \& VAssilicos, J.C. 2012 Particle image velocimetry study of fractal-generated turbulence. J. Fluid Mech. 711, 306-336.

Goto, S. \& VAssilicos, J.C. 2016 Unsteady turbulence cascades. Phys. Rev. E 94, 053108.

Grossmann, S., Lohse, D. \& ReEh, A. 1997 Different intermittency for longitudinal and transversal turbulent fluctuations. Phys. Fluids 9, 3817-3825.

HeARSt, R.J. \& LAVOIE, P. 2014 Decay of turbulence generated by a square-fractal-element grid. J. Fluid Mech. 741, 567-584.

Hurst, D. \& VAssilicos, J.C. 2007 Scalings and decay of fractal-generated turbulence. Phys. Fluids 19, 035103.

IsAzA, J.C., SAlazAR, R. \& WARhaft, Z. 2014 On grid-generated turbulence in the near- and far field regions. J. Fluid Mech. 753, 402-426.

IYER, K.P., SREENIVASAN, K.R. \& YEUnG, P.K. 2017 Reynolds number scaling of velocity increments in isotropic turbulence. Phys. Rev. E 95, 021101.

De Karman, T. \& Howarth, L. 1938 On the statistical theory of isotropic turbulence. Proc. R. Soc. Lond. A 164, 192-215.

Kitamura, T., Nagata, K., Sakai, Y., Sasoh, A., Terashima, O., Saito, H. \& Harasaki, T. 2014 On invariants in grid turbulence at moderate Reynolds numbers. J. Fluid Mech. 738, 378-406.

Kolmogorov, A.N. 1941 The local structure of turbulence in incompressible viscous fluid for very large Reynolds numbers. Dokl. Akad. Nauk SSSR 30, 301-305.

Kolmogorov, A.N. 1962 A refinement of previous hypotheses concerning the local structure of turbulence in a viscous incompressible fluid at high Reynolds number. J. Fluid Mech. 13, 82-85.

Krogstad, P.-A. \& Davidson, P.A. 2010 Is grid turbulence Saffman turbulence? J. Fluid Mech. 642, 373-394.

Krogstad, P.-Å. \& DAVIDSON, P.A. 2012 Near-field investigation of turbulence produced by multi-scale grids. Phys. Fluids 24, 035103.

Kurian, T. \& Fransson, J.H.M. 2009 Grid-generated turbulence revisited. Fluid Dyn. Res. 41, 021403.

LAIZET, S. \& LAMBALlAis, E. 2009 High-order compact schemes for incompressible flows: a simple and efficient method with quasi-spectral accuracy. J. Comput. Phys. 228 (16), 5989-6015.

Lavoie, P., DJenidi, L. \& Antonia, R.A. 2007 Effects of initial conditions in decaying turbulence generated by passive grids. J. Fluid Mech. 585, 395-420.

LELE, S.K. 1992 Compact finite difference schemes with spectral-like resolution. J. Comput. Phys. 103 (1), $16-42$.

Lumley, J.L. \& Newman, G.R. 1977 The return to isotropy of homogeneous turbulence. J. Fluid Mech. 82 (1), 161-178.

MaZellier, N. \& VASSilicos, J.C. 2010 Turbulence without Richardson-Kolmogorov cascade. Phys. Fluids 22, 075101.

Mohamed, M.S. \& Larue, J.C. 1990 The decay power law in grid-generated turbulence. J. Fluid Mech. 219, 195-214. 


\section{R. Corsini, A. Fregni, M. Spinolo and E. Stalio}

Monin, A.S. \& Yaglom, A.M. 1975 Statistical Fluid Mechanics. MIT Press.

Mora, D.O., Muñiz Pladellorens, E., Riera Turró, P., Lagauzere, M. \& Obligado, M. 2019 Energy cascades in active-grid-generated turbulent flows. Phys. Rev. Fluids 4, 104601.

NorberG, C. (Ed.) 1998 LDV-measurements in the near wake of a circular cylinder, Advances in Understanding of Bluff Body Wakes and Vortex-Induced Vibration.

Pearson, B.R., Krogstad, P.-A. \& VAN DE WAter, W. 2002 Measurements of the turbulent energy dissipation rate. Phys. Fluids 14 (3), 1288-1290.

Piller, M., Boschetto, A., Stalio, E., Schena, G. \& Errico, O. 2013 Pore-scale simulation of laminar flow through a sample of aluminum foam. J. Porous Media 16, 777-793.

Pope, S.B. 2000 Turbulent Flows. Cambridge University Press.

RotTA, J.C. 1951 Statistische theorie nichthomogener turbulenz. Z. Phys. 129 (6), 547-572.

SAFFMAN, P.G. 1967 The large-scale structure of homogeneous turbulence. J. Fluid Mech. 27, 581-593.

SEOUd, R.E. \& VASsilicos, J.C. 2007 Dissipation and decay of fractal-generated turbulence. Phys. Fluids 19, 105108.

She, Z. \& Leveque, E. 1994 Universal scaling laws in fully developed turbulence. Phys. Rev. Lett. 72, 336-339.

Simmons, L.F.G. \& SAlter, C. 1934 Experimental investigation and analysis of the velocity variations in turbulent flow. Proc. R. Soc. Lond. A 145, 212-234.

TAYLOR, G.I. 1935 Statistical theory of turbulence. Proc. R. Soc. Lond. A 151, 421-444.

Tennekes, H. \& Lumley, J.L. 1972 A First Course in Turbulence. MIT Press.

VAlente, P.C. \& VASSilicos, J.C. 2011 The decay of turbulence generated by a class of multiscale grids. J. Fluid Mech. 687, 300-340.

VAlente, P.C. \& VASSilicos, J.C. 2012 Universal dissipation scaling for nonequilibrium turbulence. Phys. Rev. Lett. 108, 214503.

VALENTE, P.C. \& VASSILICOS, J.C. 2014 The non-equilibrium region of grid-generated decaying turbulence. J. Fluid Mech. 744, 5-37.

WANG, H. \& GEORGE, W.K. 2002 The integral scale in homogeneous isotropic turbulence. J. Fluid Mech. $459,429-443$.

Zhou, T. \& AntoniA, R.A. 2000 Reynolds number dependence of the small-scale structure of grid turbulence. J. Fluid Mech. 406, 81-107. 